\title{
Non-uniform interpolatory curve subdivision with edge parameters built upon compactly supported fundamental splines
}

\author{
Carolina Vittoria Beccari · Giulio Casciola · Lucia Romani
}

Received: date / Accepted: date

\begin{abstract}
In this paper we present a family of Non-Uniform Local Interpolatory (NULI) subdivision schemes, derived from compactly supported interpolatory fundamental splines with non-uniform knots (NULIFS). For this spline family, the knot-partition is defined by a sequence of break points and by one additional knot, arbitrarily placed along each knot-interval. The resulting refinement algorithms are linear and turn out to contain a set of edge parameters that, when fixed to a value in the range [0,1], allow us to achieve special shape features by simply moving each auxiliary knot between the break points. Among all the members of this new family of schemes, we will then especially analyze the NULI 4-point refinement. This subdivision scheme has all the fundamental features of the quadratic fundamental spline basis it is originated from, namely compact support, $C^{1}$ smoothness, second order polynomials reproduction and approximation order 3. In addition the NULI 4-point subdivision algorithm has the possibility of setting consecutive edge parameters to simulate double and triple knots - that are not considered by the authors of the corresponding spline basis - thus allowing for limit curves with crease vertices, without using an ad hoc mask. Numerical examples and comparisons with other methods will be given to the aim of illustrating the performance of the NULI 4-point scheme in the case of highly non-uniform initial data.
\end{abstract}

Keywords Interpolatory subdivision · Interpolatory fundamental splines · Non-uniform knots · Centripetal parameterization $\cdot$ Edge parameters

Mathematics Subject Classification (2000) $65 \mathrm{D} 17 \cdot$ 65D07 · 65D05

\section{Introduction}

The natural way to design refinement algorithms is to conceive subdivision as a generalization of splines [5]. In this context, subdivision schemes supporting non-uniform parameterizations represent a fundamental step to make subdivision comparable to the NURBS industrial-standard. For approximating subdivision, this issue has been firstly addressed in [32] and most recently in [4,31]. In this paper we will focus on interpolatory subdivision.

Interpolatory subdivision often suffers from undesired artifacts, that arise as a consequence of the interpolation process. Usually, these effects are overcome by using Hermite-like subdivision schemes [26,29] or the so-called geometrically controlled schemes [20,28]. The first require estimating derivatives at the initial points; the second involve non-linear refinement equations. Both these techniques are shape-preserving, as they maintain convexity and monotonicity of the initial data polygon.

Although similar approaches exist in the spline context as well [10,27], it is also well-known that a properly chosen parameterization helps improving the quality of the interpolant and eliminating unwanted oscillations. In fact, a suitable parameterization does not guarantee shape preservation of the initial data, but it allows us to significantly bound the global and local deviation of the resulting curve from its data polygon [18,21,22]. In the context of interpolatory subdivision, refinement algorithms with non-uniform knots were firstly introduced by Daubechies et al. [13]. Dyn et al. [15] successively proposed to apply iterated centripetal and chordal parameterizations to the

Carolina Vittoria Beccari, Giulio Casciola

Dipartimento di Matematica, Università di Bologna, P.zza di Porta San Donato 5, 40127 Bologna, Italy

E-mail: beccari@dm.unibo.it, casciola@dm.unibo.it

Lucia Romani

Dipartimento di Matematica e Applicazioni, Università di Milano-Bicocca, Via R. Cozzi 53, 20125 Milano, Italy

E-mail: lucia.romani@unimib.it 
scheme of this family derived by up-sampling from the cubic non-uniform Lagrange-like interpolant. Since this approach requires recomputing the underlying parameterization at each subdivision step, the resulting refinement process is non-linear and, by using an ad hoc analysis, the authors prove that it generates $C^{0}$ limit curves.

In this paper we derive non-uniform, local, interpolatory subdivision algorithms by up-sampling from the general order- $n$ member of a family of Non-Uniform, Local, Interpolatory, Fundamental Splines (NULIFS). For this spline family, the knot-partition is defined by a sequence of break points and by one additional knot, arbitrarily placed along each knot-interval. As a result, the refinement rules are naturally designed to handle unequal knot intervals and to include a set of edge parameters that, when fixed to arbitrary values in the range [0,1], allow us to achieve special shape features by simply moving each auxiliary knot between the break points. This additional degree of freedom, in fact, makes it possible to generate limit curves incorporating point tension effects as well as the advantages of dealing with multiple knots.

We then present in more detail the construction of the 4-point subdivision algorithm obtained from the order3 NULIFS and we analyze its main properties. We also illustrate the 6-point scheme derived from the order-4 NULIFS. Finally we compare the NULI 4-point scheme with the NULI 6-point scheme as well as with the 4-point schemes by Daubechies et al. [13] and Dyn et al. [15]. Our analysis emphasizes that, for the purpose of applications, the NULI 4-point scheme represents a good trade-off between shape quality and computational efficiency. In fact the NULI 4-point scheme generates good quality interpolants by combining a properly chosen parameterization together with the properties of $C^{1}$-smoothness and quadratic precision. At the same time, its refinement equations are given by linear and simple formulas where the coefficients are explicitly computed depending on the edge parameter values, that can be automatically calculated or manually set to achieve a number of special features - like non-symmetric behaviors and $C^{0}$ vertices.

The remainder of the paper is organized as follows. In Section 2 we introduce the family of order- $n$ nonuniform local interpolatory fundamental splines proposed in [9] by Chui and De Villiers (Section 2.1) and we recall its distinctive features (Section 2.2); successively we discuss the issue of designing refinement equations upon the selected family members (Section 2.3). In Section 3 we present in detail the NULI 4-point scheme originated from the order-3 NULIFS (Sections 3.1 and 3.2) and analyze its main properties concerning polynomial precision, smoothness order and support width (Section 3.3). Section 4 extends the discussion to order-4 NULIFS and to the definition of the related NULI 6-point scheme. Finally, in Section 5, we show some significant examples confirming the effectiveness of our interpolatory subdivision algorithms, and especially of the NULI 4-point scheme, when applied to highly non-uniform data. A comparison with the limit curves obtained through the non-linear 4-point schemes in Dyn et al. [15] and Daubechies et al. [13] will also be presented.

\section{Towards the definition of NULI subdivision schemes}

In this section we briefly introduce the family of order- $n$ non-uniform local interpolatory fundamental splines proposed by Chui and De Villiers [9]. This family has the capability of efficiently generating optimal quality interpolants, due to its features of arbitrary knot-spacing, compact support and polynomial precision. However, for any order $n$, the quality of the NULIFS interpolant is strictly related to a suitable choice of the parameters that characterize this family member. We will therefore discuss how these parameters need to be initialized in order to generate the order- $n$ NULIFS that suits our context best. Finally we will address in great generality the issue of designing refinement equations up-on the selected family member.

\subsection{A family of Non-Uniform Local Interpolatory Fundamental Splines (NULIFS)}

Locally-supported fundamental splines leading to highly accurate local interpolation methods were firstly presented by Dahmen et al. [11]. In that seminal paper, the interpolant construction requires the solution of small linear systems with size depending on the spline degree, but not on the number of interpolation points. An explicit representation for these splines appeared only in 1990 [8]. However, this proposal was limited to the case of uniform knots. Six years later the explicit formulation of the coefficients of locally-supported interpolatory fundamental splines with arbitrary knots was provided [9].

We will now start by briefly summarizing these results, as they will be taken as a starting point for the theory developed in the remainder of the paper.

Given an ordered sequence of real values $\mathscr{X}:=\left\{x_{i}\right\}_{i \in \mathbb{Z}}$ with $\ldots<x_{i}<x_{i+1}<\ldots$, define the knot-sequence $\mathscr{T}:=\left\{t_{i}\right\}_{i \in \mathbb{Z}}$ with $\ldots<t_{i}<t_{i+1}<\ldots$ such that $t_{2 i}:=x_{i}$.

In [9] the problem of interpolation from a space of spline functions with sample points at $\mathscr{X}$ is addressed by 
the definition of a sequence of order $n \geq 3$ functions $v_{\rho, \mu, i}, i \in \mathbb{Z}$, which, for positive integers $\rho$ and $\mu$ satisfying $1 \leq \rho \leq n, 1 \leq \mu \leq \max \left\{n+\rho-3,\left\lfloor\frac{3 n}{2}\right\rfloor+\rho-5\right\}$, has the interpolation property

$$
v_{\rho, \mu, i}\left(x_{j}\right)=\delta_{i, j} \quad i, j \in \mathbb{Z},
$$

compact support

$$
\text { supp } v_{\rho, \mu, i} \subseteq \begin{cases}{\left[x_{i-\mu}, x_{i-\mu+\rho+1}\right]} & \text { for } n=3, \\ {\left[x_{i-\mu}, x_{i-\mu+\lfloor 3 n / 2\rfloor+\rho-4}\right]} & \text { for } n \geq 4,\end{cases}
$$

and generates a space $\mathscr{S}_{n, \mathscr{T}}$ of order- $n$ spline functions with knot-partition $\mathscr{T}$. Since in [9] the authors considered only the case of simple knots, the functions in $\mathscr{S}_{n, \mathscr{T}}$ are in $C^{n-2}(\mathbb{R})$. The problem of suitably defining the knotpartition as well as the influence of the free parameters $\mu$ and $\rho$ in determining the shape of the basis will be addressed in detail in Section 2.2. Hereinafter we will refer to this basis via the term NULIFS (Non-Uniform Local Interpolatory Fundamental Spline).

The NULIFS basis defines an interpolation operator

$$
\left(V_{\rho, \mu} f\right)(x)=\sum_{i \in \mathbb{Z}} f\left(x_{i}\right) v_{\rho, \mu, i}(x), \quad f \in C(\mathbb{R}),
$$

reproducing polynomials up to the order $\rho$, namely such that

$$
V_{\rho, \mu} \varphi=\varphi, \quad \forall \varphi \in \Pi_{\rho-1} .
$$

In the following, the symbol $N_{n, i}(x)$ will denote the polynomial B-spline of order $n$ with knot-partition $\mathscr{T}$ and support $\left[t_{i}, t_{i+n}\right]$. For any order $n \geq 3$, define

$$
u_{\rho, 2 \mu, i}=\sum_{j=-2 \mu}^{-2 \mu+2 \rho-1} a_{i, j} N_{n, 2 i+j}
$$

where the B-spline coefficients $a_{i, j}$ are uniquely determined by the polynomial reproduction condition (2.3) through the formula

$$
a_{i, j}=\frac{1}{(n-1) !} \frac{\sum_{k=0}^{\rho-1}(-1)^{k} \phi_{i+j, j}^{(k)}(0) \psi_{2 i+j}^{(n-1-k)}(0)}{\phi_{i+j, j}\left(x_{i}\right)} .
$$

In equation (2.5), the symbols $\psi_{i}(x)$ and $\phi_{i, j}(x)$ denote the basic polynomials

$$
\psi_{i}(x):=\prod_{q=1}^{n-1}\left(x-t_{i+q}\right)
$$

and

$$
\phi_{i, j}(x):=\prod_{j \neq q=-\mu}^{-\mu+\rho-1}\left(x-x_{i-q}\right)
$$

and the generic upper index ${ }^{(m)}$ indicates their $m$-th order derivative.

Exploiting Theorem 2.3 in [9], in case $n=3$, the compactly-supported fundamental function $v_{\rho, \mu, i}$ can be expressed in terms of the order-3 polynomial B-splines via the relation $v_{\rho, \mu, i}=u_{\rho, 2 \mu, i}$.

For $n \geq 4$, the sequence $v_{\rho, \mu, i}$ is instead defined by the formula

$$
v_{\rho, \mu, i}=\ell_{n, \tau_{n, \mu}, i}+u_{\rho, 2 \sigma_{n, \mu}, i}-\sum_{k=1}^{\lfloor n / 2\rfloor+\rho-1} u_{\rho, 2 \sigma_{n, \mu}, i}\left(x_{i-\sigma_{n, \mu}+k}\right) \ell_{n, \tau_{n, \mu}, i-\sigma_{n, \mu}+k}
$$

where

$$
\begin{gathered}
\tau_{n, \mu}:=\min \{\mu, n-3\}, \\
\sigma_{n, \mu}:=\mu-\tau_{n, \mu}+1,
\end{gathered}
$$

and 


$$
\ell_{n, v, i}(x)=\frac{\operatorname{det}\left(\tilde{A}_{n, v, i}(x)\right)}{\operatorname{det}\left(A_{n, v, i}\right)}, \quad i \in \mathbb{Z}, \quad 1 \leq v \leq n-3 .
$$

The matrices $A_{n, v, i}$ and $\tilde{A}_{n, v, i}(x)$ in (2.7) are given in terms of the B-spline sequence $N_{n, i}$ and the knots $x_{i}$ by

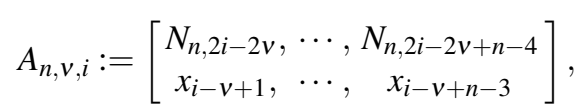

and

$$
\tilde{A}_{n, v, i}(x):=\left[\begin{array}{c}
N_{n, 2 i-2 v}, \cdots, N_{n, 2 i-2 v+n-4} \\
x_{i-v+1}, \cdots, x_{i-1}, x, x_{i+1}, \cdots, x_{i-v+n-3}
\end{array}\right] .
$$

Remark 2.1 For simplicity of notation, in the above discussion we have assumed the short symbols $N_{n, i}, v_{\rho, \mu, i}$, $u_{\rho, 2 \mu, i}, a_{i, j}, \psi_{i}, \phi_{i, j}, \ell_{n, v, i}$ in place of the corresponding extended indexing $N_{n, \mathscr{T}, i}, v_{\rho, \mu, n, \mathscr{T}, i}, u_{\rho, 2 \mu, n, \mathscr{T}, i}, a_{\rho, 2 \mu, n, \mathscr{T}, i, j}$, $\psi_{n, \mathscr{T}, i}, \phi_{\rho, 2 \mu, \mathscr{X}, i, j}, \ell_{n, v, \mathscr{T}, \mathscr{X}, i}$ that highlights the dependence on all the involved parameters.
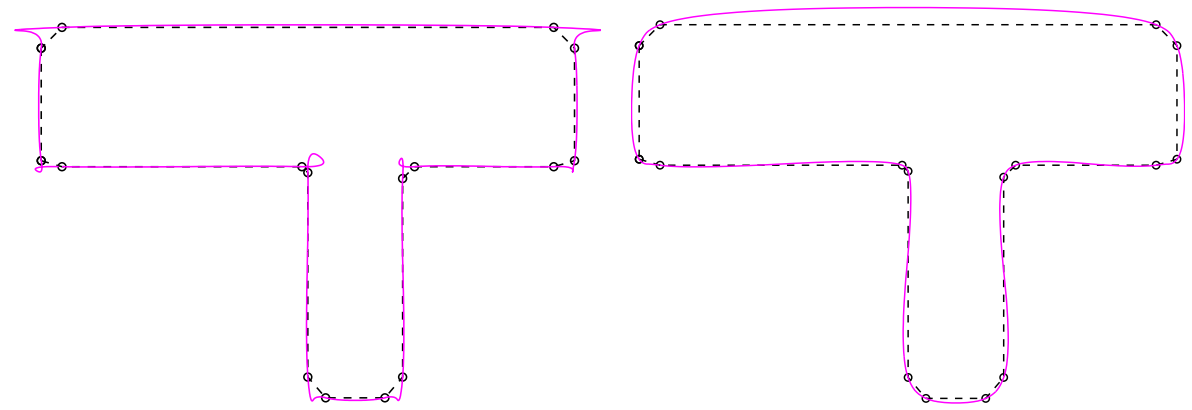

Fig. 2.1 Order-3 NULIFS interpolants of highly non-uniform data with parameters $\rho=3, \mu=2$ and uniform (left) / centripetal (right) parameterizations.

2.2 The free parameters characterizing the family members

Given a set of 2D points $P^{0}=\left\{p_{i}^{0}\right\}_{i \in \mathbb{Z}}$, the NULIFS basis $v_{\rho, \mu, i}(x)$ defines an interpolant of the form

$$
\mathscr{P}^{0}(x)=\sum_{i \in \mathbb{Z}} p_{i}^{0} v_{\rho, \mu, i}(x)
$$

Since the NULIFS basis is built upon non-uniform knots, the parameterization $\mathscr{X}=\left\{x_{i}\right\}_{i \in \mathbb{Z}}$ associated with the points $P^{0}$, weighs strongly on the quality of $\mathscr{P}^{0}(x)$. In the general context of spline interpolation, the influence of the parameterization on the quality of the interpolant, as well as the issue of determining the best parameterization for any given set of data, has been widely analyzed $[18,21,22]$. These results suggest that, computing the knotpartition according to the centripetal parameterization

$$
x_{i}=x_{i-1}+\left\|p_{i}^{0}-p_{i-1}^{0}\right\|_{2}^{\frac{1}{2}},
$$

reduces the global and local deviation of the interpolating spline from its data polygon and generates a curve that well approximates the behavior of the sample points (see Fig. 2.1). For these reasons in the following we will always assume $\mathscr{X}$ computed as in (2.9).

As discussed in the previous section, the order- $n$ NULIFS basis is also featured by the properties of $C^{n-2}$ continuity, order $\rho \leq n$ polynomials reproduction (starting from any sequence of non-equispaced samples) and compact support measured by formula (2.2). As a consequence, for any given order $n$, also the $\rho$ and $\mu$ values need to be set so as to optimize the quality of the interpolant.

Obviously, symmetry of the basis function is fundamental to generate curves that preserve symmetries of the data 
polygon. Therefore the $\mu$ value has to be chosen so that the support of the NULIFS basis becomes symmetric with respect to its central knot $x_{i}$. From (2.2) this corresponds to

$$
\mu= \begin{cases}\frac{\rho+1}{2} & \text { if } \quad n=3 \\ \frac{\lfloor 3 n / 2\rfloor+\rho-4}{2} & \text { if } n \geq 4\end{cases}
$$

In this way the NULIFS basis has the symmetric support $\left[x_{i-\mu}, x_{i+\mu}\right]$ and we avoid the undesired behaviours in Figure 2.2.
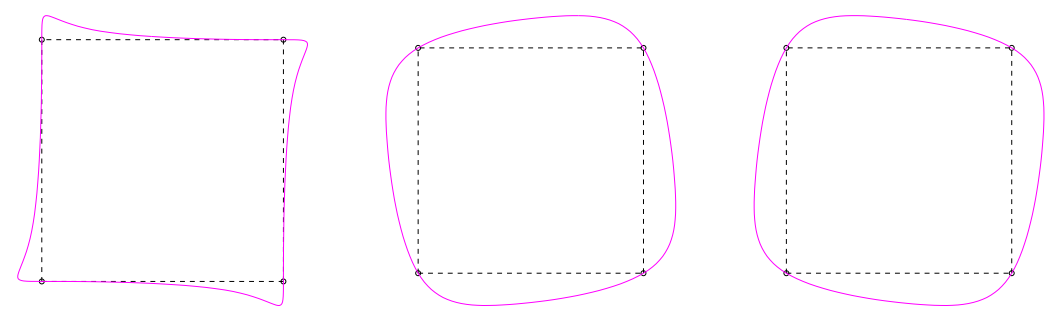

Fig. 2.2 Examples of different choices of the parameters $\rho$ and $\mu$ for order-4 NULIFS interpolants with centripetal knots: $\rho=1, \mu=2$ (left); $\rho=3, \mu=2$ (center); $\rho=3, \mu=3$ (right).

To properly decide for the value of $\rho$, we finally recall that the NULIFS basis has approximation order $\rho+1$ [11]. As a consequence, besides the choice of the parameterization, the order $\rho$ of polynomial reproduction strongly influences the overall quality of the final curve as well. To illustrate this idea, Figure 2.3 highlights how the centripetal parameterization by itself is not sufficient to guarantee a good result in terms of shape quality. It is therefore convenient to set $\rho$ at the maximum admissible value. For $n=3$, this is simply achieved by choosing $\rho=3$. Conversely, for any $n \geq 4$, the symmetry conditions (2.10) together with the requirement $\mu \in \mathbb{Z}$ do not always allow to set $\rho=n$. Hence we will choose

$$
\rho= \begin{cases}n & \text { if } \frac{\lfloor 3 n / 2\rfloor+n-4}{2} \in \mathbb{Z} \\ n-1 & \text { otherwise }\end{cases}
$$

Based on this discussion, in the remainder of the paper we will always define the knot-partition $\mathscr{X}$ according to the centripetal parameterization (2.9) and, for any given order $n$, we will always assume the parameters $\mu$ and $\rho$ to be set as in equations (2.10) and (2.11). Notice however that, although the initial parameterization is fixed, we still have one degree of freedom per each edge $\overline{p_{i}^{0} p_{i+1}^{0}}$, which is given by the possibility of arbitrarily placing the additional knot $t_{2 i+1}$ inside the interval $] x_{i}, x_{i+1}\left[\right.$. Different choices of $t_{2 i+1}$ give rise to different shapes, as illustrated in Figure 2.4. In the following sections, whenever not specified, we will assume the knot parameter $t_{2 i+1}$ to be placed at $\frac{x_{i}+x_{i+1}}{2}$.
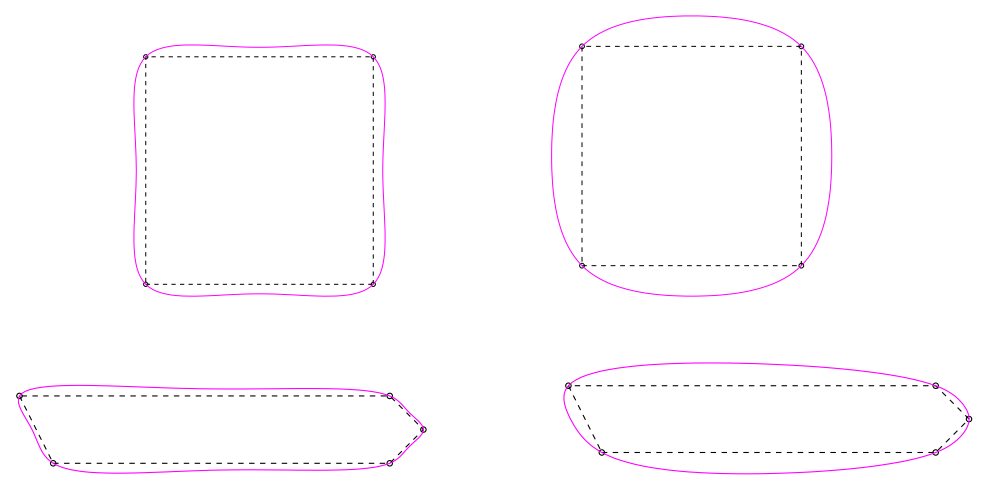

Fig. 2.3 Examples of different choices of the parameters $\rho$ and $\mu$ for order-4 NULIFS interpolants with centripetal knots: $\rho=2, \mu=2$ (left); $\rho=4, \mu=3$ (right). 

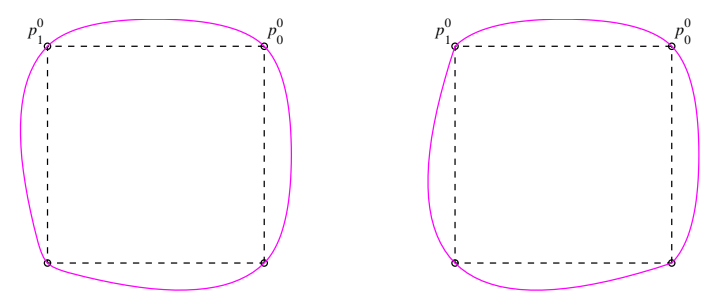

Fig. 2.4 Order-3 NULIFS interpolants with $\rho=3, \mu=2$, centripetal parameterization and knot-partition $\mathscr{T}=$ $\{0,0.125,0.25,0.475,0.5,0.525,0.75,0.875,1\}$ (left), $\mathscr{T}=\{0,0.125,0.24,0.25,0.5,0.75,0.76,0.875,1\}$ (right).

\subsection{The design of non-uniform local interpolatory (NULI) refinement equations}

The original idea of designing an interpolating subdivision process by up-sampling from an analytic interpolant defined on an irregular grid was presented by Daubechies et al. [13]. The refinement procedure consists in keeping points corresponding to the even grid values and inserting a new point in correspondence to odd grid values.

Let $P^{0}=\left\{p_{i}^{0}\right\}_{i \in \mathbb{Z}}$ be the initial set of points and $\mathscr{X}$ the associated non-uniform parameterization in (2.9). Let also $\Lambda=\left\{\lambda_{i}\right\}_{i \in \mathbb{Z}}$ be an initial set of parameters such that $\left.\lambda_{i} \in\right] 0,1[$ is associated with the $i$-th edge.

The knot-partition $\mathscr{T}=\left\{t_{i}\right\}_{i \in \mathbb{Z}}$ is defined by

$$
t_{2 i}:=x_{i}, \quad t_{2 i+1}:=x_{i}+\lambda_{i}\left(x_{i+1}-x_{i}\right)
$$

Suppose now that the initial sequence $\mathscr{X}$ is iteratively refined by inserting a new knot in correspondence to the midpoint of each parameter interval, and let $\mathscr{X}^{k}$ be the $k$-times refined knot-partition. In analogy with this notation, $\mathscr{T}^{k}$ is computed from $\mathscr{X}^{k}$ through equation (2.12). We will also denote by $\Lambda^{k}$ the set of edge parameters after $k$ iterations.

At the $k$-th iteration, the NULIFS basis determines an interpolant to the data $P^{k}$ of the form

$$
\mathscr{P}^{k}(x)=\sum_{i \in \mathbb{Z}} p_{i}^{k} v_{\rho, \mu, i}(x)
$$

The refinement rules of the NULI scheme are then defined by

$$
\begin{aligned}
p_{2 i}^{k+1} & =p_{i}^{k} \\
p_{2 i+1}^{k+1} & =\mathscr{P}^{k}\left(x_{2 i+1}^{k+1}\right) .
\end{aligned}
$$

The point evaluation in the second line of (2.14) depends, according to (2.13), on the availability of the bi-infinite sequence $\left\{p_{i}^{k}\right\}_{i \in \mathbb{Z}}$. To obtain closed limit curves, any given (finite) initial sequence $p_{0}^{0}, \cdots, p_{N}^{0}$ should be extended periodically to yield the bi-infinite initial sequence $\left\{p_{i}^{0}\right\}_{i \in \mathbb{Z}}$, and so forth for each successive level $k>0$.

Remark 2.2 The NULI subdivision scheme generates limit curves that, with respect to the order- $n$ NULIFS interpolant, represent a tighter fitting of the given data (see Fig. 5.2). In fact, the subdivision scheme defines a sequence of refined polylines where the newly inserted vertices belong to the NULIFS interpolants of denser and denser nested sets of points, that naturally represent a closer approximation of the underlying data polygons.

Denote now by $d_{i}$ the length of the knot-interval with endpoints $x_{i}, x_{i+1}$, namely

$$
d_{i}=x_{i+1}-x_{i}
$$

The refinement of the underlying knot-partition implies that, at each successive iteration, the knot-intervals are updated according to the formula

$$
d_{2 i}^{k+1}=d_{2 i+1}^{k+1}=\frac{d_{i}^{k}}{2}, \quad \text { where } \quad k \geq 0 \quad \text { and } \quad d_{i}^{0}:=d_{i}
$$

At the same time, at the $k$-th iteration, the edge parameters $\Lambda^{k}$ are computed from $\Lambda^{0}$ according to any suitable method. One of the possible strategies will be discussed in Section 3. 
Remark 2.3 Relations (2.16) imply that the extra knot per interval, that is defined in (2.12) and is used to generate the $k$-th level NULIFS interpolant, will not be inserted in the next subdivision step. In fact, due to (2.14), a new point is inserted by evaluating the NULIFS interpolant at the mid points of the parameter intervals and thus the knot-partition should be accordingly refined through (2.16).

We can now formulate the NULIFS basis with knots $\mathscr{T}^{k}$ in terms of the knot-intervals $d_{i}^{k}$ and of the edge parameters $\lambda_{i}^{k}$. Since the NULIFS basis is invariant under linear transformation of the knot-vector, the NULIFS interpolant depends only on the local configuration of the knot-intervals. Thus, by combining the second row of (2.14) with the definition of $\mathscr{P}^{k}$ in (2.13), the newly inserted point can be expressed as

$$
p_{2 i+1}^{k+1}=\sum_{h=0}^{2 \mu-1} c_{h}\left(d_{i-\mu+1}^{k}, \ldots, d_{i+\mu-1}^{k}, \lambda_{i-\mu+2}^{k}, \ldots, \lambda_{i+\mu-2}^{k}\right) p_{i}^{k},
$$

where the coefficients $c_{h}, h=0, \ldots, 2 \mu-1$ are given by the values of the NULIFS basis functions at the knot $x_{2 i+1}^{k+1}$. In our setting, the order- $n$ NULIFS basis functions have symmetric support of width $2 \mu$, so that, for any $k \geq 0$, we will have $2 \mu$ refinement coefficients in the interval $] x_{i}^{k}, x_{i+1}^{k}[$. More precisely, due to the compact support of the NULIFS basis, the refinement coefficients depend on the knot intervals $d_{i-\mu+1}^{k}, \ldots, d_{i+\mu-1}^{k}$ and edge parameters $\lambda_{i-\mu+2}^{k}, \ldots, \lambda_{i+\mu-2}^{k}$, which explains the notation $c_{h}\left(d_{i-\mu+1}^{k}, \ldots, d_{i+\mu-1}^{k}, \lambda_{i-\mu+2}^{k}, \ldots, \lambda_{i+\mu-2}^{k}\right)$.

In the following sections we will see that the proposed general method allows us to explicitly derive the set of refinement coefficients that defines a $2 \mu$-point subdivision scheme.

By definition, for each order $n$, the resulting subdivision schemes reproduce polynomials up to the order $\rho$. Thus, recalling the discussion in Section 2.2, these subdivision schemes reproduce polynomials up to the order $n$ or $n-1$ (see e.g. Proposition 3.1).

The knot-intervals configuration for the $k$-th and $(k+1)$-th subdivision steps is shown in Figure 2.5. Observe that, after a few refinements, the scheme tends to become uniform everywhere except that in the neighborhood of the initial points. This gives rise to a piecewise-uniform subdivision scheme [16,23].

Remark 2.4 In the proposed strategy, the non-uniform parameters do not need to be recomputed at each refinement step, since they are simply updated through relations (2.16). However, this is not the only possible approach. Another option is to iteratively recompute the parameterization at each refinement step, as proposed in [15]. While the former method allows us to keep the scheme linear, the latter gives rise to a non-linear refinement process. Our numerical testing showed that the two methods produce similar results, thus there is no significant advantage in using the non-linear approach.

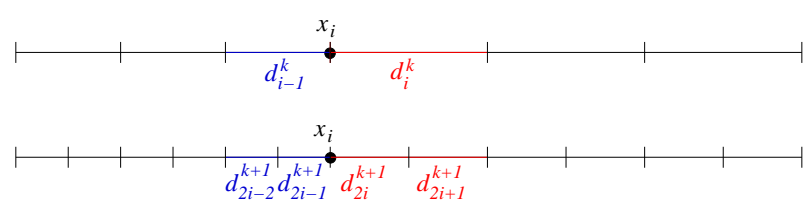

Fig. 2.5 Knot-intervals configuration in the neighborhood of the initial knot $x_{i}$ after $k$ and $k+1$ iterations of the NULI subdivision scheme.

Although the above approach holds for each order $n \geq 3$, in practice, only 4 or 6-point refinements are usually considered well-suited for applications. In our setting, these schemes are originated by the order-3 and 4 NULIFS basis respectively. Hence, in the following, we will analyze these two cases in more detail.

\section{NULI 4-point subdivision built upon order-3 NULIFS}

In this section we present the NULI 4-point scheme originated from the order-3 NULIFS. As a fundamental step towards the definition of this subdivision scheme, we start out by deriving an explicit representation of the class of order-3 non-uniform local interpolatory fundamental splines. We then provide the related refinement algorithm and analyze its main properties concerning polynomial precision, smoothness order and support width. 


\subsection{An explicit representation of the order-3 NULIFS basis}

Recalling the general parameters setting strategy discussed in Section 2.2, we will consider the NULIFS basis $v_{3,2, i}(x)$ (i.e. with $n=3, \rho=3$ and $\mu=2$ ), with knot-partition (2.12).

¿From relation (2.2), supp $v_{3,2, i}(x) \subseteq\left[x_{i-2}, x_{i+2}\right]$ and in this span we have the related knot-intervals $d_{j}=$ $x_{j+1}-x_{j}$ and the corresponding edge parameters $\lambda_{j}, j=i-2, \ldots, i+1$.

Formula (2.4) provides the expression of the NULIFS basis $v_{3,2, i}(x)$ centered at $x_{i}$ and satisfying the interpolatory condition (2.1), i.e.

$$
v_{3,2, i}(x)=\sum_{j=-4}^{1} a_{i, j} N_{3,2 i+j}(x) .
$$

As discussed in Section 2.3, without loss of generality we can now assume $x_{i}=0$. In this way the coefficients $a_{i, j}$ are given by

$$
\begin{array}{ll}
a_{i,-4}=\frac{\left(d_{i-2}\right)^{2}\left(\lambda_{i-2}-1\right)}{2 d_{i-1}\left(d_{i-2}+d_{i-1}\right)}, & a_{i,-3}=\frac{d_{i-2} \lambda_{i-1}}{2\left(d_{i-2}+d_{i-1}\right)},
\end{array}
$$

More precisely, the order-3 basis function $v_{3,2, i}(x)$ in (3.1), is made of the 8 quadratic polynomial pieces $v_{3,2, i}^{h}(x)$, $h=1, \ldots, 8$, that reparameterized in the interval $\left[0, d_{i}\right]$, are described by the following equations:

$$
\begin{aligned}
v_{3,2, i}^{1}(x) & =\frac{\left(1+\lambda_{i}\right) x-2 d_{i} \lambda_{i}}{2 \lambda_{i} d_{i-1}\left(d_{i-1}+d_{i}\right)} x \\
v_{3,2, i}^{3}(x) & =1+\left(\frac{1}{d_{i-1}}-\frac{1}{d_{i}}\right) x-\frac{\left(d_{i-1}+d_{i}+d_{i+1}+\left(d_{i}+d_{i+1}-d_{i-1}\right) \lambda_{i}\right)}{2 \lambda_{i} d_{i-1} d_{i}\left(d_{i}+d_{i+1}\right)} x^{2} \\
v_{3,2, i}^{5}(x) & =\frac{\left(d_{i-1}+d_{i}\right)\left(1-\lambda_{i}\right) x+d_{i+1}\left(2 d_{i-1} \lambda_{i}+\left(1+\lambda_{i}\right) x\right)}{2 \lambda_{i} d_{i} d_{i+1}\left(d_{i-1}+d_{i}\right)} x \\
v_{3,2, i}^{7}(x) & =\frac{\left(\lambda_{i}-1\right)}{2 \lambda_{i} d_{i+1}\left(d_{i}+d_{i+1}\right)} x^{2}
\end{aligned}
$$

for $x \in\left[0, \lambda_{i} d_{i}\right]$ (see equation (2.12)) and

$$
\begin{aligned}
& v_{3,2, i}^{2}(x)=-\frac{\lambda_{i}}{2\left(1-\lambda_{i}\right) d_{i-1}\left(d_{i-1}+d_{i}\right)}\left(d_{i}-x\right)^{2}, \\
& v_{3,2, i}^{4}(x)=\frac{\left(d_{i+1}+d_{i}\right) \lambda_{i}\left(d_{i}-x\right)+d_{i-1}\left(2 d_{i+1}\left(1-\lambda_{i}\right)+\left(2-\lambda_{i}\right)\left(d_{i}-x\right)\right)}{2\left(1-\lambda_{i}\right) d_{i} d_{i-1}\left(d_{i+1}+d_{i}\right)}\left(d_{i}-x\right), \\
& v_{3,2, i}^{6}(x)=1+\left(\frac{1}{d_{i+1}}-\frac{1}{d_{i}}\right)\left(d_{i}-x\right)-\frac{\left(d_{i+1}+d_{i}+d_{i-1}+\left(d_{i}+d_{i-1}-d_{i+1}\right)\left(1-\lambda_{i}\right)\right)}{2\left(1-\lambda_{i}\right) d_{i+1} d_{i}\left(d_{i}+d_{i-1}\right)}\left(d_{i}-x\right)^{2}, \\
& v_{3,2, i}^{8}(x)=\frac{\left(2-\lambda_{i}\right)\left(d_{i}-x\right)-2 d_{i}\left(1-\lambda_{i}\right)}{2\left(1-\lambda_{i}\right) d_{i+1}\left(d_{i+1}+d_{i}\right)}\left(d_{i}-x\right),
\end{aligned}
$$

for $x \in\left[\lambda_{i} d_{i}, d_{i}\right]$.

A plot of these quadratic polynomial pieces is represented in Figure 3.1 for both the uniform and non-uniform cases, assuming $\lambda_{i}=\frac{1}{2}, \forall i$.

Remark 3.1 Notice that the expressions of $v_{3,2, i}^{2}, v_{3,2, i}^{4}, v_{3,2, i}^{6}, v_{3,2, i}^{8}$ in (3.3) can be obtained by applying to $v_{3,2, i}^{7}$, $v_{3,2, i}^{5}, v_{3,2, i}^{3}, v_{3,2, i}^{1}$ in (3.2) the transformation

$$
x \rightarrow d_{i}-x, \quad \lambda_{i} \rightarrow 1-\lambda_{i}, \quad d_{i-1} \rightarrow d_{i+1}, \quad d_{i+1} \rightarrow d_{i-1} .
$$

Relations (3.2) and (3.3) allow us to easily evaluate the NULIFS basis at any arbitrary point $x$, provided that the knot-intervals $d_{i-1}, d_{i}, d_{i+1}$ and the edge parameter $\lambda_{i}$ are given. This is a key ingredient in the definition of the interpolating scheme proposed in the following section. 

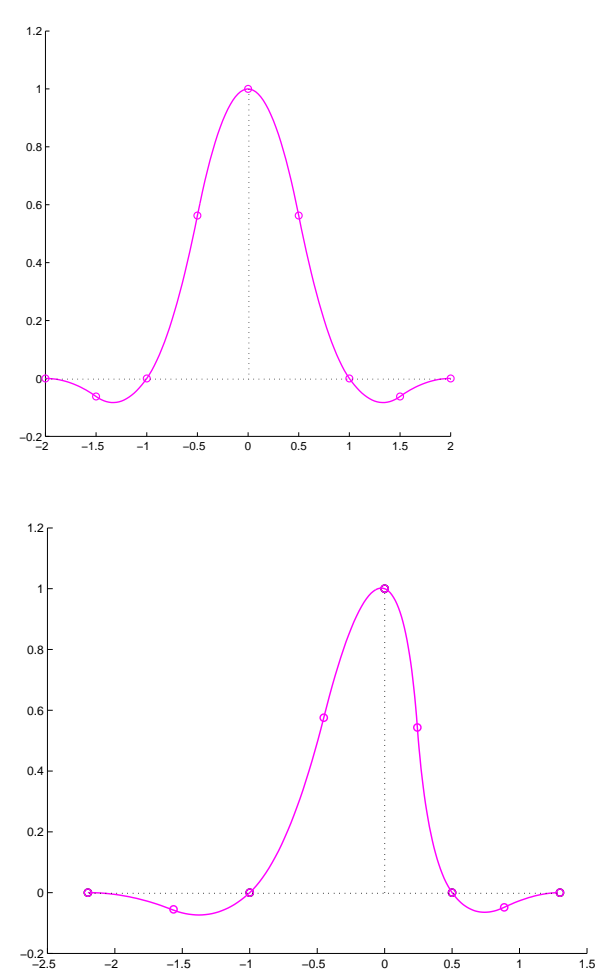
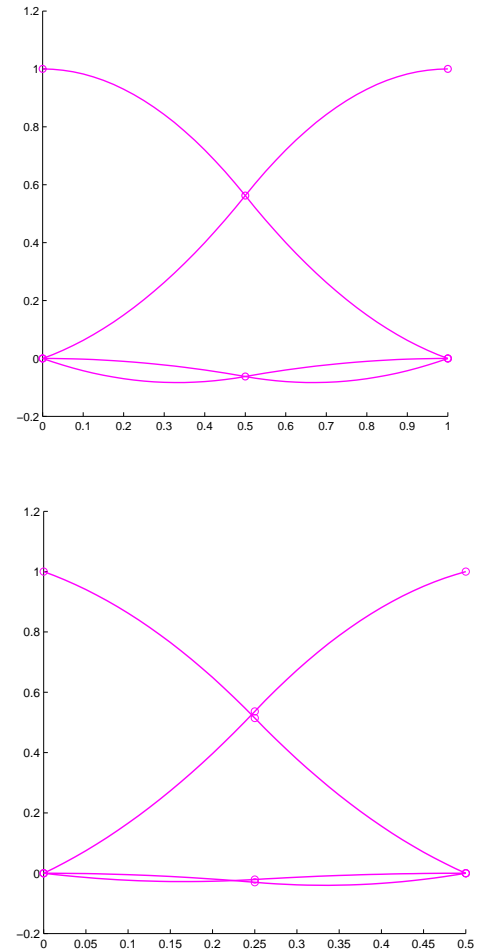

Fig. 3.1 First row: the 8 polynomial pieces of the order- 3 NULIFS basis function with uniform knots $\left\{x_{i-2}, x_{i-1}, x_{i}, x_{i+1}, x_{i+2}\right\}=$ $\{-2,-1,0,1,2\}$. Second row: the 8 polynomial pieces of the order-3 NULIFS basis function with non-uniform knots $\left\{x_{i-2}, x_{i-1}, x_{i}, x_{i+1}, x_{i+2}\right\}=\{-2.2,-1,0,0.5,1.3\}$ (left) and the 8 pieces of the same basis function in the interval $[0,0.5]$ (right).

\subsection{The NULI 4-point refinement algorithm}

Let us denote by $P^{0}=\left\{p_{i}^{0}\right\}_{i \in \mathbb{Z}}$ the initial polyline, by $\Lambda^{0}=\left\{\lambda_{i}^{0}\right\}_{i \in \mathbb{Z}}$ the edge parameters and by $\mathscr{X}=\left\{x_{i}\right\}_{i \in \mathbb{Z}}$ the associated knots in (2.9). Exploiting formula (2.15) for all $i$, we work out the initial knot-intervals $d_{i}^{0}$.

At each subdivision level $k$ we also need a set of edge parameters $\Lambda^{k}$. In principle these parameters can be chosen arbitrarily, i.e. either manually specified at each refinement step or automatically computed starting from the initial set $\Lambda^{0}$ by any iterative updating strategy.

However, as previously discussed, these parameters derive by construction from the structure of the NULIFS basis, which includes one auxiliary knot per each knot-interval. In particular, the parameter $\lambda_{i}$ moves the auxiliary knot $t_{2 i+1}$ between the two endpoints of the interval $] x_{i}, x_{i+1}$ [ (see equation (2.12)).

Thus the strategy that we will use in the following aims at obtaining a limit curve that simulates the shape of the NULIFS interpolant of the initial data, with edge parameters $\Lambda^{0}$.

At subdivision level $k=0$ the edge parameter $\lambda_{i}^{0}$ is assigned to the edge $\overline{p_{i}^{0} p_{i+1}^{0}}$. Moreover, if $\lambda_{i}^{0} \neq \frac{1}{2}$, either one or both endpoints of such edge are tagged. At the successive step $(k=1)$, the edge $\overline{p_{i}^{0} p_{i+1}^{0}}$ is split into the two edges $\overline{p_{2 i}^{1} p_{2 i+1}^{1}}$ and $\overline{p_{2 i+1}^{1} p_{2 i+2}^{1}}$. Each of these new edges inherit the edge parameter value $\lambda_{i}^{0}$ if one of its vertices is tagged, otherwise the edge parameter is set to the value $\frac{1}{2}$ (Fig. 3.2). This edge parameters updating method generates a linear subdivision process.

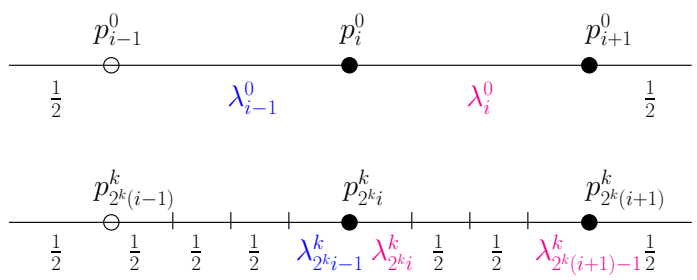

Fig. 3.2 Initial edge parameters and their configuration at the $k$-refinement step where $\lambda_{2^{k}{ }_{i-1}}^{k}=\lambda_{i-1}^{0}$ and $\lambda_{2^{k} i}^{k}=\lambda_{2^{k}(i+1)-1}^{k}=\lambda_{i}^{0}$. Filled circles correspond to initially tagged vertices. 
At the general refinement level $k$, we will thus have the four points $p_{i-1}^{k}, p_{i}^{k}, p_{i+1}^{k}, p_{i+2}^{k}$, the corresponding knotintervals $d_{i-1}^{k}, d_{i}^{k}, d_{i+1}^{k}$, computed according to (2.16), and the edge parameters $\lambda_{i-1}^{k}, \lambda_{i}^{k}, \lambda_{i+1}^{k}$ derived following the above method.

According to (2.12), the interval $d_{i}^{k}$ is split into two sub-intervals, one to the left and one to the right of the additional knot. Thus, if $\left.\lambda_{i}^{k} \in\right] 0, \frac{1}{2}$ [, we substitute the value $x=d_{i}^{k} / 2$ into equations (3.3), while if $\lambda_{i}^{k} \in\left[\frac{1}{2}, 1[\right.$ we substitute it into (3.2). In this way, for all $k \geq 0$, we get the coefficients $c_{h}:=c_{h}\left(d_{i-1}^{k}, d_{i}^{k}, d_{i+1}^{k}, \lambda_{i}^{k}\right), h=0, \ldots, 3$ where

$$
\begin{aligned}
c_{0} & =\frac{\lambda_{i}^{k}\left(d_{i}^{k}\right)^{2}}{8\left(\lambda_{i}^{k}-1\right) d_{i-1}^{k}\left(d_{i-1}^{k}+d_{i}^{k}\right)}, \\
c_{1} & =\frac{\lambda_{i}^{k}\left[-d_{i}^{k} d_{i+1}^{k}+d_{i}^{k} d_{i-1}^{k}+4 d_{i+1}^{k} d_{i-1}^{k}-\left(d_{i}^{k}\right)^{2}\right]-2 d_{i-1}^{k}\left(d_{i}^{k}+2 d_{i+1}^{k}\right)}{8\left(\lambda_{i}^{k}-1\right) d_{i-1}^{k}\left(d_{i}^{k}+d_{i+1}^{k}\right)}, \\
c_{2} & =\frac{\lambda_{i}^{k}\left[3\left(d_{i}^{k}\right)^{2}+5 d_{i}^{k} d_{i+1}^{k}+3 d_{i}^{k} d_{i-1}^{k}+4 d_{i+1}^{k} d_{i-1}^{k}\right]-2\left(d_{i-1}^{k}+d_{i}^{k}\right)\left(d_{i}^{k}+2 d_{i+1}^{k}\right)}{8\left(\lambda_{i}^{k}-1\right) d_{i+1}^{k}\left(d_{i-1}^{k}+d_{i}^{k}\right)}, \\
c_{3} & =\frac{\left(2-3 \lambda_{i}^{k}\right)\left(d_{i}^{k}\right)^{2}}{8\left(\lambda_{i}^{k}-1\right) d_{i+1}^{k}\left(d_{i}^{k}+d_{i+1}^{k}\right)},
\end{aligned}
$$

if $\left.\lambda_{i}^{k} \in\right] 0, \frac{1}{2}[$ and

$$
\begin{aligned}
& c_{0}=\frac{\left(1-3 \lambda_{i}^{k}\right)\left(d_{i}^{k}\right)^{2}}{8 \lambda_{i}^{k} d_{i-1}^{k}\left(d_{i}^{k}+d_{i-1}^{k}\right)}, \\
& c_{1}=\frac{\left(\lambda_{i}^{k}-1\right)\left[3\left(d_{i}^{k}\right)^{2}+5 d_{i}^{k} d_{i-1}^{k}+3 d_{i}^{k} d_{i+1}^{k}+4 d_{i-1}^{k} d_{i+1}^{k}\right]+2\left(d_{i+1}^{k}+d_{i}^{k}\right)\left(d_{i}^{k}+2 d_{i-1}^{k}\right)}{8 \lambda_{i}^{k} d_{i-1}^{k}\left(d_{i+1}^{k}+d_{i}^{k}\right)}, \\
& c_{2}=\frac{\left(\lambda_{i}^{k}-1\right)\left[-d_{i}^{k} d_{i-1}^{k}+d_{i}^{k} d_{i+1}^{k}+4 d_{i-1}^{k} d_{i+1}^{k}-\left(d_{i}^{k}\right)^{2}\right]+2 d_{i+1}^{k}\left(d_{i}^{k}+2 d_{i-1}^{k}\right)}{8 \lambda_{i}^{k} d_{i+1}^{k}\left(d_{i}^{k}+d_{i-1}^{k}\right)}, \\
& c_{3}=\frac{\left(\lambda_{i}^{k}-1\right)\left(d_{i}^{k}\right)^{2}}{8 \lambda_{i}^{k} d_{i+1}^{k}\left(d_{i+1}^{k}+d_{i}^{k}\right)},
\end{aligned}
$$

if $\lambda_{i}^{k} \in\left[\frac{1}{2}, 1[\right.$.

Notice that the set of coefficients $c_{0}, c_{1}, c_{2}, c_{3}$ in (3.6) can also be obtained by applying the transformation (3.4) to the coefficients $c_{3}, c_{2}, c_{1}, c_{0}$ in (3.5).

We observe now that equations (3.5) and (3.6) are still well-defined if $\lambda_{i}^{k} \in[0,1]$, namely including the endpoints of the interval. Thus, differently than in [9], in the refinement equations of the NULI subdivision schemes, the knot $t_{2 i+1}$ may coincide either with $x_{i}$ or $x_{i+1}$, simulating a double knot in the knot-partition $\mathscr{T}$. In addition, if we initially set the parameters of two successive edges to the values $\lambda_{i-1}^{0}=1$ and $\lambda_{i}^{0}=0$, which corresponds to a triple knot in the knot-partition $\mathscr{T}$, we will get a crease vertex in correspondence to the point $p_{i}^{0}$ (see Proposition 3.3). Thus, in the remainder of the paper, we will assume the edge parameters to be set in $[0,1]$. The corresponding refinement process is summarized by the following algorithm.

\section{Algorithm 1:}

Given an initial polyline $P^{0}=\left\{p_{i}^{0}\right\}_{i \in \mathbb{Z}}$ :

1. compute the initial knot-intervals $d_{i}^{0}$ as follows:

1.1 compute the knot sequence $\mathscr{X}$ according to (2.9), thus $x_{i}=x_{i-1}+\left\|p_{i}^{0}-p_{i-1}^{0}\right\|_{2}^{\frac{1}{2}}$;

1.2 define the initial knot-intervals $d_{i}^{0}$ through (2.15), namely $d_{i}^{0}=x_{i+1}-x_{i}$;

2. assign to each initial edge a parameter $\lambda_{i}^{0} \in[0,1]$;

3. $\forall k \geq 0$ :

3.1 for each edge $\overline{p_{i}^{k} p_{i+1}^{k}}$

3.1 .1 if $\lambda_{i}^{k} \in\left[0,1 / 2\left[\right.\right.$ compute the coefficients $c_{h}, h=0, \ldots, 3$ from (3.5);

3.1.2 else if $\lambda_{i}^{k} \in[1 / 2,1]$ compute the coefficients $c_{h}, h=0, \ldots, 3$ from (3.6); 
3.2 refine the polyline as

$$
\begin{aligned}
& p_{2 i}^{k+1}=p_{i}^{k}, \\
& p_{2 i+1}^{k+1}=c_{0} p_{i-1}^{k}+c_{1} p_{i}^{k}+c_{2} p_{i+1}^{k}+c_{3} p_{i+2}^{k} ;
\end{aligned}
$$

3.3 update the knot-intervals through

$$
d_{2 i}^{k+1}=d_{2 i+1}^{k+1}=\frac{d_{i}^{k}}{2}
$$

3.4 update the edge parameters $\lambda_{i}^{k}$ following the method discussed at the beginning of this section (see Fig. $3.2)$.

Remark 3.2 The NULI 4-point scheme in (3.7) can be seen as an extension of Dubuc's 4-point scheme to the non-uniform case. In fact, when all $d_{i}^{0}$ are equal (i.e. in the case of uniform parameterization) and $\lambda_{i}^{0}=\frac{1}{2}$ for all $i$, in equations (3.5) and (3.6) we have the coefficients $c_{0}=c_{3}=-\frac{1}{16}, c_{1}=c_{2}=\frac{9}{16}$, which yield the uniform 4-point scheme [14]. On the other hand, Dubuc's 4-point scheme can be obtained by fitting a cubic polynomial to four equispaced points and evaluating at the midpoint of the central interval. Hence, in the uniform case, the two construction methods are equivalent because the values of the four fundamental Lagrange polynomials of degree 3 at $\frac{1}{2}$ coincide with the values of $\left\{v_{3,2,0}\left(j-\frac{1}{2}\right)\right\}_{j=-1, \ldots, 2}$, and so in this case the NULI 4-point scheme reproduces polynomials up to degree 3 (and not merely degree 2 ) like Dubuc's 4-point scheme.

\subsection{Analysis of the NULI 4-point scheme}

In this section we show that the NULI 4-point scheme inherits all the properties of the quadratic NULIFS basis on which it was built upon, namely quadratic precision, approximation order $3, C^{1}$-smoothness and local-support.

Proposition 3.1 [Polynomial precision] For any initial set of edge parameters $\Lambda^{0}$, the NULI 4-point scheme reproduces the set $\Pi_{2}$ of polynomials up to degree 2 whenever applied to any sequence of arbitrarily spaced samples. In addition, the NULI 4-point scheme reproduces the set $\Pi_{3}$ of polynomials up to degree 3 whenever applied to evenly-spaced samples and $\lambda_{i}^{0}=\frac{1}{2}, \forall i$.

Proof: When all knots are simple, quadratic precision follows directly by construction of the considered scheme: in fact, at each step, we upsample from the order-3 NULIFS interpolant (see equation (2.14)), which reproduces quadratic polynomials ${ }^{1}$. However, to prove polynomial reproduction for any possible parameter configuration, including $\lambda_{i}^{0}=0,1$, we can follow a more general strategy. For any subdivision level $k$, consider the quadruple of values $\left(x_{j}^{k},\left(x_{j}^{k}\right)^{h}\right), h=0,1,2, j=i-1, \ldots, i+2$, that are non uniform samples of a constant, linear or quadratic function. If we now apply the equations (3.5) or (3.6) to the 4 considered points, it can be easily verified that, for any value of $\lambda_{i}^{k} \in[0,1]$, the inserted point, associated with the parameter $\frac{1}{2}\left(x_{i}^{k}+x_{i+1}^{k}\right)$ has the expression $\left(\frac{1}{2}\left(x_{i}^{k}+x_{i+1}^{k}\right)\right)^{h}$.

Cubic precision in the uniform setting follows from the fact that the refinement rules (3.7) reduce to Dubuc's 4-point scheme (see Remark 3.2).

It was proved that, under certain conditions [24,25], the exactness of a non-uniform subdivision scheme for polynomials up to degree $m$, is necessary and sufficient for achieving an approximation order $m+1$ for any function which is smooth enough. Thus the following result holds.

Corollary 3.1 (Approximation order) The NULI 4-point scheme has approximation order 3.

To analyze the smoothness of the NULI 4-point scheme we observe that, after a few rounds of subdivision, the knot intervals assume a piecewise-uniform configuration of the kind ..., 1, 1, $\alpha, \alpha, \alpha$, ... where $\alpha>0$ (see Fig. 2.5). Analogously, in these regions, the edge parameters assume the common value $\lambda_{i}^{k}=1 / 2$ (see Fig. 3.2). As a consequence, away from the vertex identified by the junction point of these two uniform knot sequences, the NULI 4-point scheme brings back to the uniform 4-point scheme [14], which is known to be $C^{1}$. Thus, we only need to analyze the smoothness of the scheme in the regions surrounding the junction points.

\footnotetext{
1 The property of quadratic reproduction refers to the scalar case. Thus, to have such property, if the samples are arbitrarily taken on a quadratic polynomial, the parameters should be the corresponding abscissae.
} 
Binary refinements defined over irregular knot sequences that are halved at each step were already analyzed in [33]. For the NULI 4-point scheme the situation is slightly different, since, in the neighborhood of the junction points, we need to take into account both the local knot intervals $d_{i}^{k}$-s and the edge parameters $\lambda_{i}^{k}$-s. The following analysis relies on the generalizations of the results in [33]. Notice also that the vertex corresponding to the junction point might be tagged or not, as discussed in Section 3.2. To the purpose of our analysis, it is sufficient to consider the situation of a tagged vertex, as the local subdivision matrix in the case of a non-tagged vertex is just a particular case of the latter with $\lambda_{i}^{k}=\frac{1}{2}$.

Proposition 3.2 (Smoothness order) The NULI 4-point scheme generates $C^{1}$-continuous limit curves for any choice of initial knots $\mathscr{X}=\left\{x_{i}\right\}_{i \in \mathbb{Z}}$ and edge parameters $\Lambda=\left\{\lambda_{i}^{0}\right\}_{i \in \mathbb{Z}}$, such that the edge parameters of two subsequent initial edges do not assume at the same time the values $\lambda_{i-1}^{0}=1$ and $\lambda_{i}^{0}=0$.

Proof: Without loss of generality, we will assume the junction point of the two regular knot sequences obtained after $k>2$ subdivision steps to be $x_{i}=0$. From the above discussion, the NULI 4-point scheme is $C^{1}$-continuous everywhere except at the point $x_{i}=0$. Moreover, in the neighborhood of the junction point $x_{i}=0$, the local subdivision matrix of the NULI 4-point scheme has the structure

$$
M=\left[\begin{array}{ccccccc}
-\frac{1}{16} & \frac{9}{16} & \frac{9}{16} & -\frac{1}{16} & 0 & 0 & 0 \\
0 & 0 & 1 & 0 & 0 & 0 & 0 \\
0 & c_{0}\left(1,1, \alpha, \lambda_{i-1}^{k}\right) & c_{1}\left(1,1, \alpha, \lambda_{i-1}^{k}\right) & c_{2}\left(1,1, \alpha, \lambda_{i-1}^{k}\right) & c_{3}\left(1,1, \alpha, \lambda_{i-1}^{k}\right) & 0 & 0 \\
0 & 0 & 0 & 1 & 0 & 0 & 0 \\
0 & 0 & c_{0}\left(1, \alpha, \alpha, \lambda_{i}^{k}\right) & c_{1}\left(1, \alpha, \alpha, \lambda_{i}^{k}\right) & c_{2}\left(1, \alpha, \alpha, \lambda_{i}^{k}\right) & c_{3}\left(1, \alpha, \alpha, \lambda_{i}^{k}\right) & 0 \\
0 & 0 & 0 & 0 & 1 & 0 & 0 \\
0 & 0 & 0 & -\frac{1}{16} & \frac{9}{16} & \frac{9}{16} & -\frac{1}{16}
\end{array}\right],
$$

where the coefficients $c_{h}\left(1,1, \alpha, \lambda_{i-1}^{k}\right), h=0, \ldots, 3$, are computed by formulas (3.5) or (3.6), depending on the value of $\lambda_{i-1}^{k}$ and $c_{h}\left(1, \alpha, \alpha, \lambda_{i}^{k}\right), h=0, \ldots, 3$ are computed analogously according to the value of $\lambda_{i}^{k}$.

Using the symbolic computation program Mathematica, it is easy to verify that the $7 \times 7$ local subdivision matrix in (3.8) has eigenvalues $\ell_{0}=1, \ell_{1}=\frac{1}{2}$, and all the remaining eigenvalues $\ell_{j}$, with $j=2, \cdots, 6$, are either scalar with modulus smaller than $\frac{1}{2}$ or defined by a symbolic expression depending only on the parameters $\alpha, \lambda_{i-1}^{k}$ and $\lambda_{i}^{k}$. Solving the symbolic inequalities obtained by imposing the conditions

$$
\left|\ell_{j}\right|<\frac{1}{2} \quad \forall j \geq 2 \text { such that } \ell_{j} \text { is symbolic, }
$$

it can be verified that (3.9) holds for any values of $\alpha, \lambda_{i-1}^{k}$ and $\lambda_{i}^{k}$ in their spans of definition, i.e. $\alpha>0$ and $\lambda_{i-1}^{k}, \lambda_{i}^{k} \in[0,1]$ (excluding the case $\lambda_{i-1}^{k}=1$ and at the same time $\lambda_{i}^{k}=0$ ). Thus, for any possible initial edge parameters configuration - except for the case $\lambda_{i-1}^{0}=1$ and contemporaneously $\lambda_{i}^{0}=0$ - the eigenvalues of $M$ satisfy the necessary $C^{1}$ conditions.

Notice also that, due to the parameters updating strategy proposed in Section 3.2 the scheme is stationary, namely the same refinement matrix $M$ is applied at each iteration around the point $x_{i}$. As a consequence, for each eigenvalue $\ell_{j}$ of $M$ with eigenvector $v_{j}$, the basis function $\mathscr{F}_{v_{j}}$ of the scheme satisfies

$$
\ell_{j} \mathscr{F}_{v_{j}}(h)=\mathscr{F}_{v_{j}}\left(\frac{h}{2}\right) .
$$

Now, repeating the procedure in [33], it can be verified that, if the scheme satisfies relation (3.10) and the two leading eigenvectors reproduce 1 and $t$ - which is true by Proposition 3.1 - the conditions $\ell_{0}=1, \ell_{1}=\frac{1}{2},\left|\ell_{j}\right|<$ $\frac{1}{2} \forall j \geq 2$ are also sufficient to guarantee $C^{1}$-smoothness of the scheme.

Proposition 3.3 For any choice of initial knots $\mathscr{X}=\left\{x_{i}\right\}_{i \in \mathbb{Z}}$, if the edge parameters of two subsequent edges are set to the values $\lambda_{i-1}^{0}=1$ and $\lambda_{i}^{0}=0$, the limit curve of the NULI 4-point scheme is $C^{0}$-continuous at the point $p_{i}^{0}$.

Proof: The local subdivision matrix $M$ in (3.8), corresponding to the configuration $\lambda_{i-1}^{0}=1, \lambda_{i}^{0}=0$, generates the eigenvalues $\ell_{0}=1, \ell_{1}=\ell_{2}=\frac{1}{2},\left|\ell_{j}\right|<\frac{1}{2} \forall j \geq 3$. In this case the eigenvalues $\ell_{1}$ and $\ell_{2}$ have two linearly independent eigenvectors, causing the scheme to be $C^{0}$ at the junction point $x_{i}=0$ (Fig. 3.4 right). 
Remark 3.3 Notice that the authors of the order-3 NULIFS basis [9] did not consider the case of double and triple knots. Conversely, with the NULI 4-point scheme we handle also those configurations and our analysis shows that the limit curves are $C^{0}$-continuous in the case of a triple knot configuration and $C^{1}$-continuous otherwise.

Proposition 3.4 (Support width) The basis function $\mathscr{F}_{e_{i}}(h)$ of the NULI 4-point scheme centered at $x_{i}$ has compact support $s=\left[x_{i-3}, x_{i+3}\right]$.

Proof: The basis function $\mathscr{F}_{i}(h)$ is obtained as the limit function of the scheme applied to the vector $e_{i}$, whose $i$ th entry is one with the remaining entries being zero.

At refinement step $k=0$, the basis function $\mathscr{F}_{i}(h)$ vanishes outside the interval $s^{0}=\left[x_{i-2}, x_{i+2}\right]$. At each successive step $k>0$, the width of the support is extended by a factor $\frac{x_{i-2}-x_{i-3}}{2^{k}}$ and $\frac{x_{i+3}-x_{i+2}}{2^{k}}$ on its left and right hand side respectively. Thus, after $N$ steps we will have

$$
s^{N}=\left[x_{i-2}-\sum_{k=1}^{N} \frac{x_{i-2}-x_{i-3}}{2^{k}}, x_{i+2}+\sum_{k=1}^{N} \frac{x_{i+3}-x_{i+2}}{2^{k}}\right] .
$$

Therefore, $s=\lim _{N \rightarrow+\infty} s^{N}=\left[x_{i-3}, x_{i+3}\right]$.

A plot of the basis function $\mathscr{F}_{e_{i}}(h)$ is shown in Figs. 3.3 and 3.4.

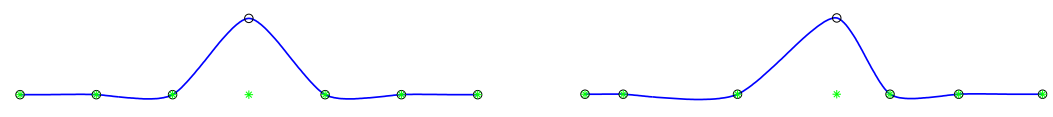

Fig. 3.3 Basis function of the NULI 4-point scheme with uniform knots (left) and non-uniform knots (right). The edge parameters are set to $\lambda_{i}^{0}=\frac{1}{2}, \forall i$.

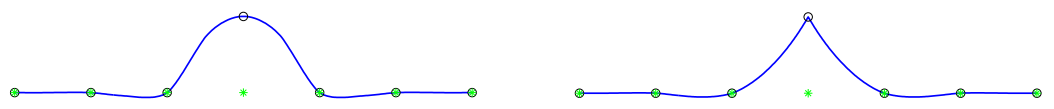

Fig. 3.4 Basis function of the NULI 4-point scheme with uniform knots, edge parameters $\lambda_{i-1}^{0}=0.1, \lambda_{i}^{0}=0.9($ left $) / \lambda_{i-1}^{0}=1, \lambda_{i}^{0}=0$ (right) and tagged vertex $\left(x_{i}, 1\right)$.

\section{The order-4 NULIFS basis and the NULI 6-point scheme}

Exploiting formula (2.6), the NULIFS basis $v_{4,3, i}(x)$ centered at $x_{i}$ and satisfying the interpolatory condition (2.1), is defined by

$$
v_{4,3, i}(x)=\sum_{j=-6}^{2} a_{i, j} N_{4,2 i+j}(x),
$$

where the coefficients $a_{i, j}$ are given in Appendix.

Remark 4.1 In the case of uniform parameterization and mid-point additional knots (i.e. $\lambda_{i}=\frac{1}{2}, \forall i$ ), the order-4 NULIFS basis $v_{4,3, i}(x)$ with the extended knot-partition (2.12) becomes the $C^{2}$ LICS (Local Interpolatory Cardinal Spline) basis $[8,6]$. Also, the uniform order-4 NULIFS basis $v_{2,2, i}(x)$ coincides with the $C^{2} \mathrm{~B} 2$-spline proposed by Chu [7]. 
Observe that, from relation (2.2), $\operatorname{supp}_{4,3, i}(x) \subseteq\left[x_{i-3}, x_{i+3}\right]$ and consequently the coefficients $a_{i, j}$ of the order-4 NULIFS basis $v_{4,3, i}(x)$ enclose the six parameters $d_{j}, j=i-3, \ldots, i+2$. In this way, the coefficients $c_{h}, h=0, \ldots, 5$, defining the NULI 6-point insertion rule, depend on the parameters $d_{i-2}^{k}, d_{i-1}^{k}, d_{i}^{k}, d_{i+1}^{k}, d_{i+2}^{k}$ and on the edge parameters $\lambda_{i-1}^{k}, \lambda_{i}^{k}, \lambda_{i+1}^{k}$, namely $c_{h}=c_{h}\left(d_{i-2}^{k}, d_{i-1}^{k}, d_{i}^{k}, d_{i+1}^{k}, d_{i+2}^{k}, \lambda_{i-1}^{k}, \lambda_{i}^{k}, \lambda_{i+1}^{k}\right)$. For the sake of conciseness, we will not provide a formulation analogous to (3.5) for the coefficients of the NULI 6-point scheme. In fact, although their explicit expressions can be symbolically derived by repeating the computations described in Section 2.3 , they turn out to be quite long.

Moreover, following the same method proposed in Proposition 3.2, it can be proved that the NULI 6-point scheme generates $C^{2}$ limit curves. In particular, the subdivision scheme corresponding to the NULI 6-point, generates $C^{2}$ limit curves in any uniform region. In addition, in the neighborhood of the junction point between two non-uniform regions, the local subdivision matrix associated to the scheme has eigenvalues $\ell_{0}=1, \ell_{1}=\frac{1}{2}, \ell_{2}=\frac{1}{4},\left|\ell_{j}\right|<\frac{1}{4}$ $\forall j \geq 3$.

The main advantage of the NULI 6-point scheme over its 4-point counterpart is given by higher smoothness as well as higher degree of polynomial precision. However, it is worsened by wider support and by the fact that evaluating its coefficients requires more computational effort. These disadvantages limit its importance in applications and prevent any possibility of extension to the surface case.

\section{Application examples and comparisons}

In this section we show some application examples to demonstrate that the NULI 4-point scheme represents a good trade-off between computational efficiency and shape quality. To this aim we will first compare the NULI 4-point scheme with the corresponding order-3 NULIFS interpolant and successively with the NULI 6-point scheme and the non-linear subdivision schemes by Dyn et al. [15] and Daubechies et al. [13].

For interpretation of the references to color in the figures, the reader is referred to the web-version of this paper. We also let the reader notice that the different quality of the curves in the following examples appears more evident by enlarging the pdf format of this manuscript.

Figures 5.1 and 5.2 illustrate the results we got by applying the NULI 4-point scheme to highly non-uniformly spaced initial data.
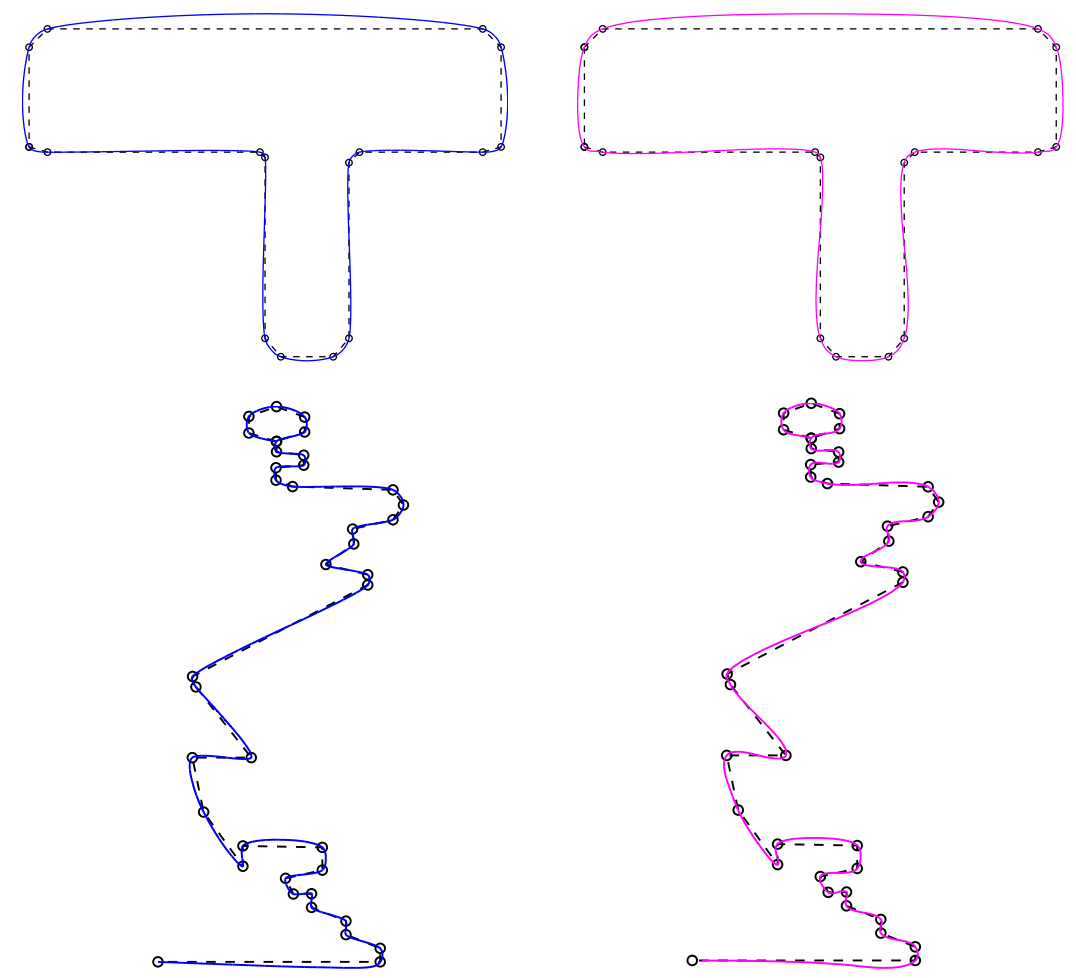

Fig. 5.1 The NULI 4-point limit curve (left/blue) and the NULIFS quadratic interpolant (right/magenta). 

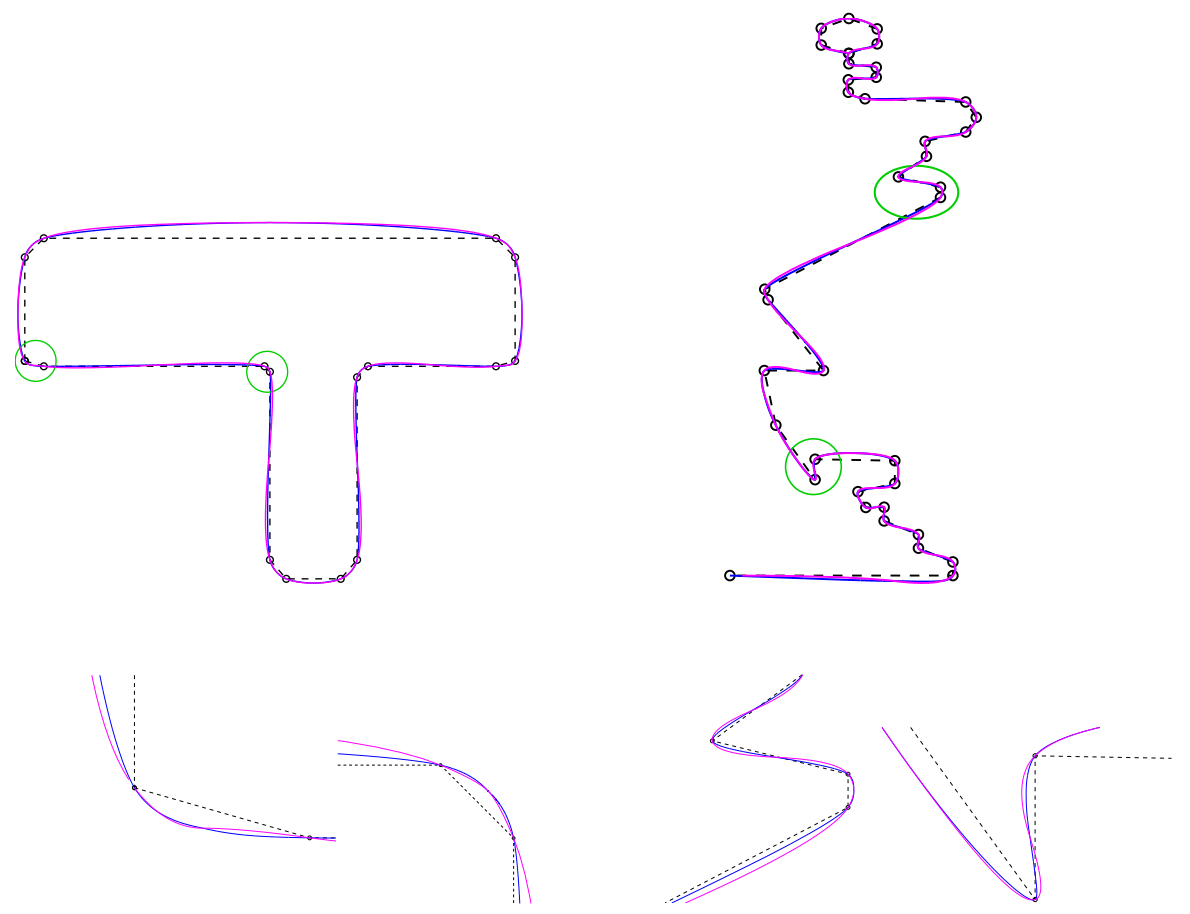

Fig. 5.2 First row: comparison of the interpolation curves in Fig. 5.1. Second row: enlargement of two details in the above interpolation curves The NULI 4-point limit curve is in color blue, while the NULIFS quadratic interpolant in magenta.
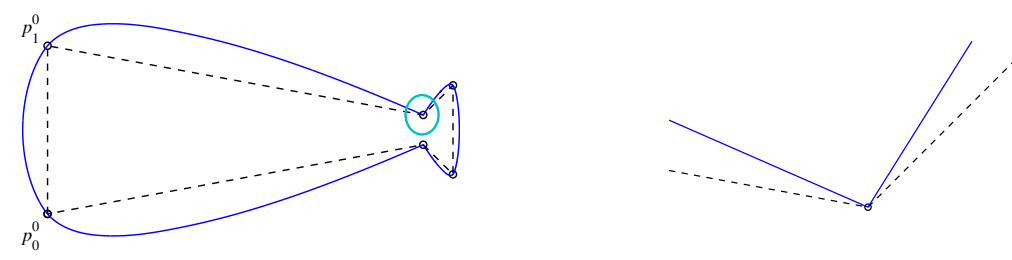

Fig. 5.3 The NULI 4-point limit curve obtained with edge parameters $\lambda_{1}^{0}=1, \lambda_{2}^{0}=0, \lambda_{4}^{0}=1, \lambda_{5}^{0}=0$ and tagged vertices $p_{2}^{0}, p_{5}^{0}$ (left) and enlargement of one detail (right).

As discussed in Section 2.3 (see Remark 2.2), if compared with the corresponding order-3 NULIFS interpolant, the limit curve of the NULI 4-point scheme turns out to be tighter to the initial data polygon (in the sense of Remark 2.2).

Although there are many proposals of stationary and non-stationary subdivision schemes whose refinement equations include shape parameters $[1-3,12,17,19,30]$, the authors are not aware of any existing scheme whose parameters set has a behavior comparable to the NULI 4-point scheme. In fact, so far parameters have been introduced either to control the tension of the limit curve [2,3,30], to increase its smoothness [17,19] or to reproduce salient curves $[1,3,12,30]$. Differently, the edge parameters of the NULI 4-point scheme are used to simulate the behavior of double and triple knots. Thus no comparison with other subdivision schemes including parameters will be presented in the case of $\lambda_{i}^{0} \mathrm{~s}$ different from $\frac{1}{2}$.

Figures 5.3 and 5.4 show some of the shape effects that can be achieved by playing with different values of the edge parameters $\lambda_{i}^{0}$. Differently than the corresponding order-3 NULIFS interpolant, the NULI 4-point scheme is capable of generating crease vertices (Fig. 5.3). Figure 5.4 compares the behavior of the NULI 4-point limit curves, with the parameters updating strategy discussed in Section 3.2, with the order-3 NULIFS interpolant, when starting from the same initial edge parameters.

Figures 5.5 and 5.6 (left) illustrate limit curves obtained through the NULI 6-point scheme and the corresponding order-4 NULIFS interpolant.

Finally, Figures 5.6 (right) and 5.7 present a comparison of the NULI 4-point interpolant with the limit curves obtained through the NULI 6-point scheme and the non-linear 4-point schemes by Dyn et al. [15] and Daubechies 

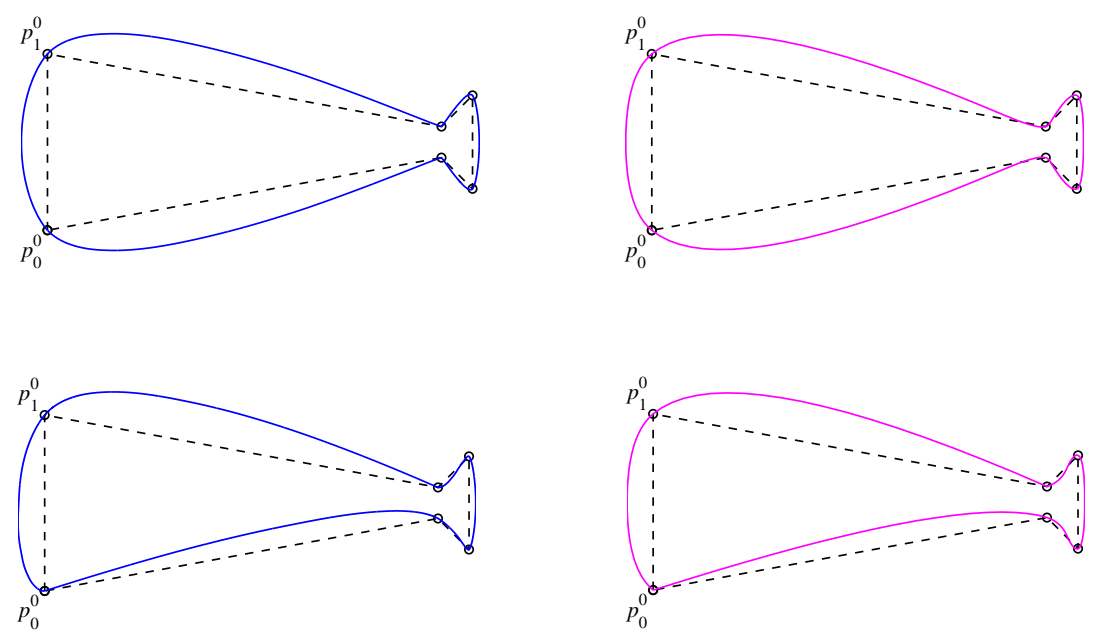

Fig. 5.4 The NULI 4-point limit curve (left/blue) and the NULIFS quadratic interpolant (right/magenta). Top row: tagged vertices $p_{2}^{0}, p_{5}^{0}$ and edge parameters $\lambda_{1}^{0}=0.9, \lambda_{2}^{0}=0.1, \lambda_{4}^{0}=0.9, \lambda_{5}^{0}=0.1$; bottom row: tagged vertices $p_{2}^{0}, p_{0}^{0}$ and edge parameters $\lambda_{1}^{0}=1, \lambda_{5}^{0}=1$.

et al. [13].

All these numerical examples indicate that when $\lambda_{i}^{0}=\frac{1}{2}$, the NULI 4-point scheme generates similar curves with respect to the ones obtained through such schemes.
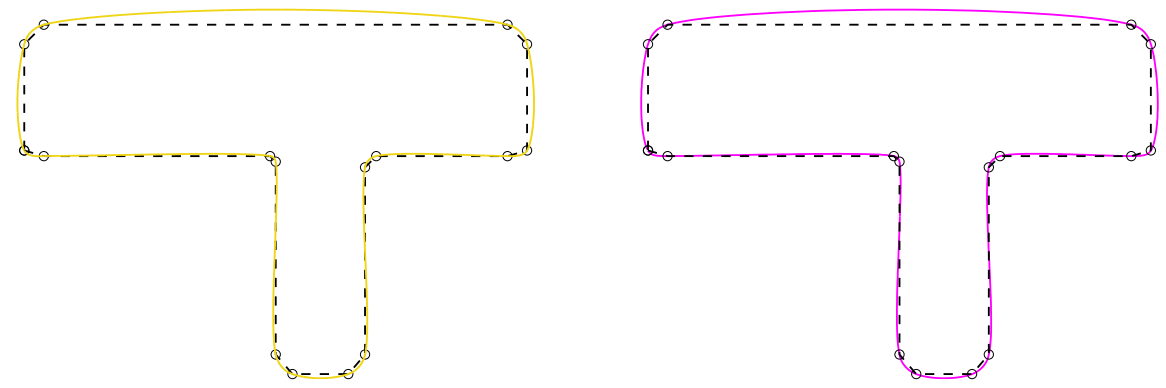

Fig. 5.5 The NULI 6-point limit curve (left/yellow) and the NULIFS cubic interpolant (right/magenta).

\section{Conclusions and future work}

We have presented a family of non-uniform interpolating subdivision schemes originated from order- $n$ locally supported fundamental splines with arbitrary knots. The resulting refinement algorithms are linear and, besides exploiting the advantages of a centripetal parameterization to interpolate non-uniformly spaced data, they include a set of edge parameters that allow great shape flexibility.

Among all schemes that can be derived in the proposed way, we have addressed in detail the construction of the NULI 4 and 6-point schemes.

Our analysis emphasizes that, to the purpose of applications, the NULI 4-point scheme turns out to be the optimal member of its family of schemes and also advantageous with respect to other non uniform 4-point schemes. In fact, if compared with the non-linear 4-point schemes by Dyn et al. [15] or Daubechies et al. [13], it has the advantage of being linear, so that we were able to prove convergence and smoothness results, and computationally cheaper, since the parameterization is not recomputed at each step. On the other hand, although its NULI 6-point counterpart 


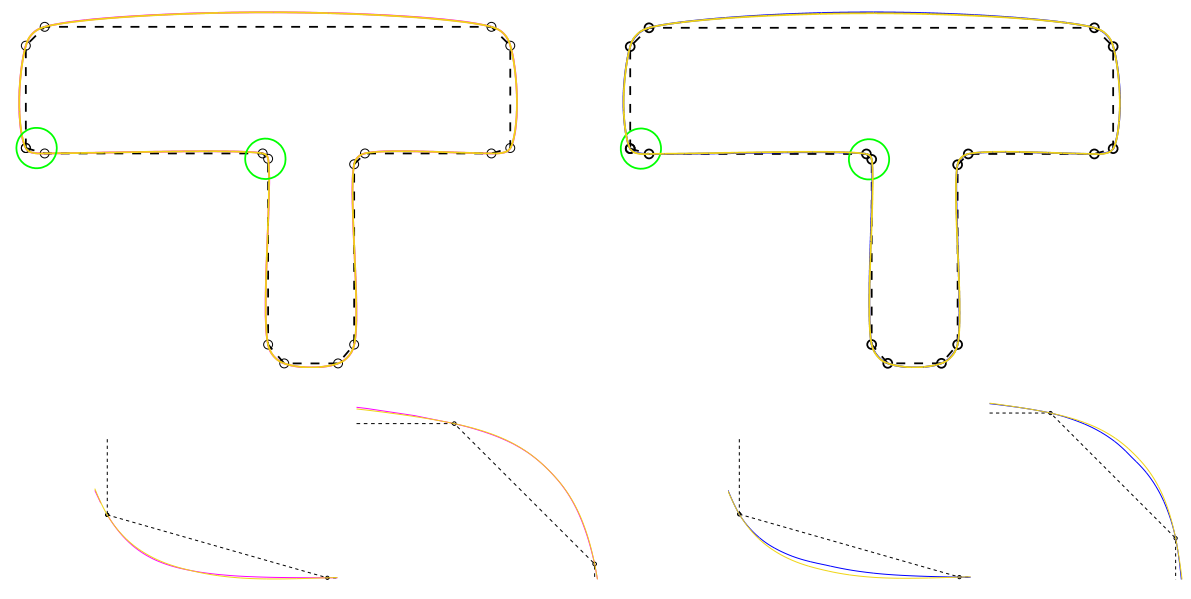

Fig. 5.6 First row: comparison of the NULI 6-point limit curve with the cubic NULIFS interpolant (left) and with the NULI 4-point limit curve (right). Second row: enlargement of two details in the above interpolation curves. The NULI 4 and 6-point limit curves are respectively in colors blue and yellow. The cubic NULIFS interpolant is in color magenta.

generates $C^{2}$ limit curves, the NULI 4-point scheme is computationally advantageous, as the coefficients of the refinement equations are described by much simpler formulas. Finally, with respect to both the aforementioned schemes, the NULI 4-point scheme generates limit curves with similar shapes whenever $\lambda_{i}^{0}=\frac{1}{2}, \forall i$. In addition, for different values of $\lambda_{i}^{0} \in[0,1]$, the NULI 4-point scheme allows us to achieve special shape features like point tension effects or $C^{0}$ vertices that cannot be obtained by the NULIFS basis considered in [9].

For all its desirable properties, the NULI 4-point refinement algorithm constitutes a key ingredient towards the definition of non-uniform, local, interpolatory subdivision surfaces over meshes of arbitrary topology.

Acknowledgements This research was supported by the University of Bologna and the University of Milano-Bicocca, Italy.

The authors are grateful to the anonymous referees for their useful suggestions.

\section{References}

1. C. Beccari, G. Casciola, L. Romani: A non-stationary uniform tension controlled interpolating 4-point scheme reproducing conics. Comput. Aided Geom. Design 24(1), 1-9 (2007)

2. C. Beccari, G. Casciola, L. Romani: An interpolating 4-point ternary non-stationary subdivision scheme with tension control. Comput. Aided Geom. Design 24(4), 210-219 (2007)

3. C. Beccari, G. Casciola, L. Romani: Shape controlled interpolatory ternary subdivision. Appl. Math. Comput. 215(3), 916-927 (2009)

4. T.J. Cashman, N.A. Dodgson, M.A. Sabin: A symmetric, non-uniform, refine and smooth subdivision algorithm for general degree Bsplines. Comput. Aided Geom. Design 26(1), 94-104 (2009)

5. G.M. Chaikin: An algorithm for high speed curve generation. Comput. Graphics Image Process. 3, 346-349 (1974)

6. J.J. Chen, A.K. Chan, C.K. Chui: A local interpolatory cardinal spline method for the determination of eigenstates in quantum-well structures with arbitrary potential profiles. IEEE J. Quantum Electronics 30(2), 269-274 (1994)

7. K.-C. Chu: B3-splines for interactive curve and surface fitting. Comput. Graphics 14(2), 281-288 (1990)

8. C.K. Chui: Vertex splines and their applications to interpolation of discrete data. In: W. Dahmen, M. Gasca and C.A. Micchelli (Eds.), Computation of Curves and Surfaces, 1990, pp. 137-181.

9. C.K. Chui, J.M. De Villiers: Applications of optimally local interpolation to interpolatory approximants and compactly supported wavelets. Math. Comp. 65(213), 99-114 (1996)

10. C. Conti, R. Morandi: Piecewise $C^{1}$-shape-preserving Hermite interpolation. Computing 56(4), 323-341 (1996)

11. W. Dahmen, T.N.T. Goodman, C.A. Micchelli: Compactly supported fundamental functions for spline interpolation. Numer. Math. 52, 639-664 (1988)

12. S. Daniel, P. Shunmugaraj: An approximating $C^{2}$ non-stationary subdivision scheme. Comput. Aided Geom. Design 26(7), 810-821 (2009)

13. I. Daubechies, I. Guskov, W. Sweldens: Regularity of irregular subdivision. Constr. Approx. 15(3), 381-426 (1999)

14. S. Dubuc: Interpolation through an iterative scheme. J. Math. Anal. Appl. 114(1), 185-204 (1986)

15. N. Dyn, M. Floater, K. Hormann: Four-point curve subdivision based on iterated chordal and centripetal parameterizations. Comput. Aided Geom. Design 26(3), 279-286 (2009)

16. N. Dyn, J.A. Gregory, D. Levin: Piecewise uniform subdivision schemes. In: M. Dæhlen, T. Lyche, and L.L. Schumaker (Eds.), Mathematical Methods for Curves and Surfaces, pp.111-120, Vanderbilt University Press, Nashville (TN), (1995) 

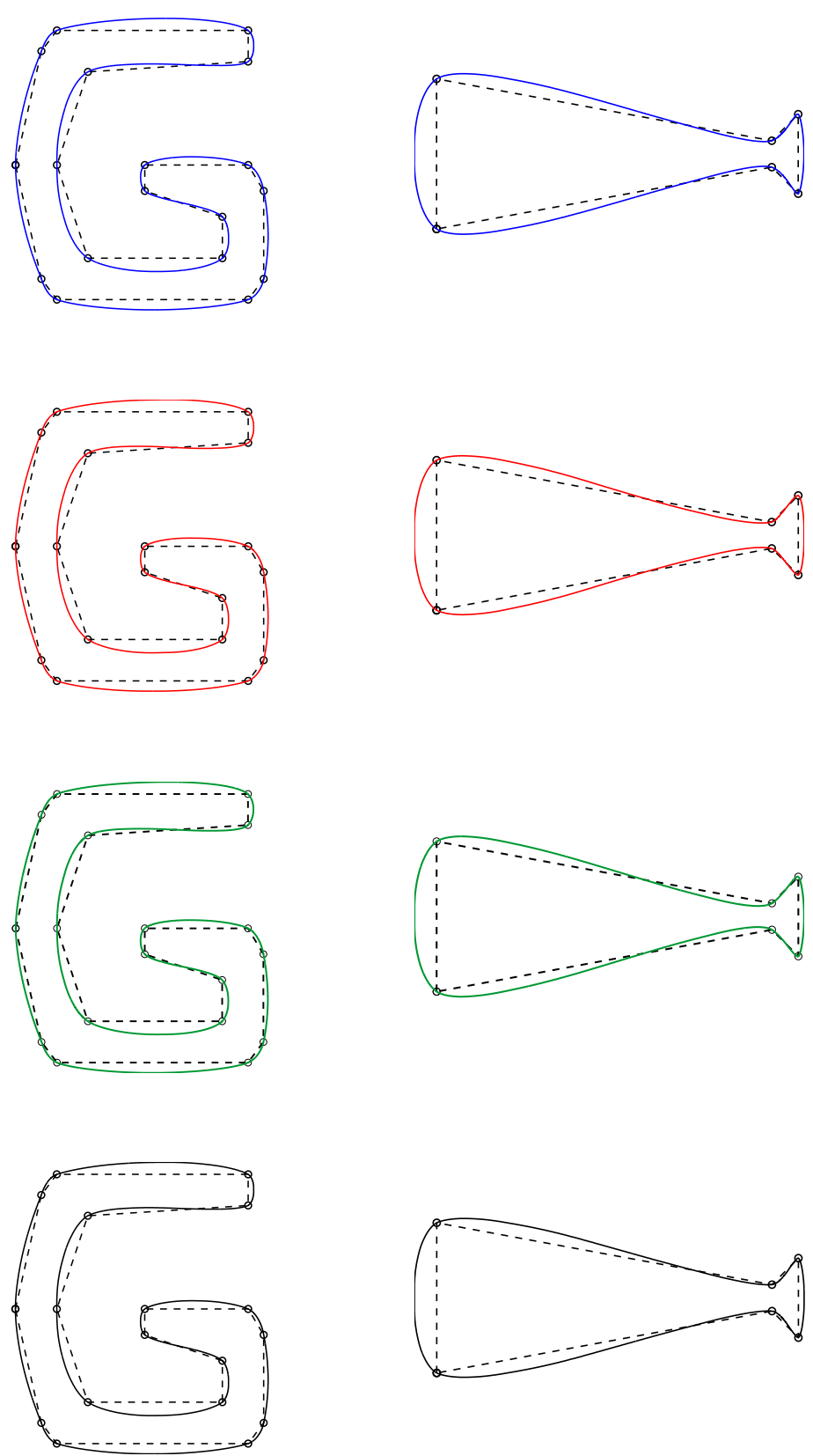

Fig. 5.7 Limit curves obtained through the NULI 4-point (first row/blue), the non-linear 4-point scheme by Dyn et al. [15] (second row/red), the non-uniform 4-point by Daubechies et al. with centripetal parameterization [13] (third row/green) and the non-linear NULI 4-point scheme with iterated centripetal parameterizations (fourth row/black).

17. N. Dyn, D. Levin, C.A. Micchelli: Using parameters to increase smoothness of curves and surfaces generated by subdivision. Comput. Aided Geom. Design 7, 129-140 (1990)

18. M. Floater: On the deviation of a parametric cubic spline interpolant from its data polygon. Comput. Aided Geom. Design 25, 148-156 (2008)

19. M.F. Hassan, I.P. Ivrissimitzis, N.A. Dodgson, M.A. Sabin: An interpolating 4-point $C^{2}$ ternary stationary subdivision scheme. Comput. Aided Geom. Design 19(1), 1-18 (2002)

20. F. Kuijt, R. van Damme: Shape preserving interpolatory subdivision schemes for non uniform data. J. Approx. Theory 114(1), 1-32 (2002)

21. E.B. Kuznetsov, A.Yu. Yakimovich: The best parameterization for parametric interpolation. J. Comput. Appl. Math. 191(2), 239-245 (2006)

22. E.T.Y. Lee: Choosing nodes in parametric curve interpolation. Computer-Aided Design 21(6), 363-370 (1989)

23. A. Levin: Combined subdivision schemes. PhD thesis, Tel-Aviv university (2000)

24. A. Levin: Polynomial generation and quasi-interpolation in stationary non-uniform subdivision. Comput. Aided Geom. Design 20(1), 41-60 (2003)

25. A. Levin: The importance of polynomial reproduction in piecewise uniform subdivision. In: R. Martin, H. Bez and M.A. Sabin (Eds.), Mathematics of Surfaces XI, Proceedings of 11th IMA international Conference, pp. 272-307, Springer (2005) 
26. T. Lyche, J.-L. Merrien: $C^{1}$ interpolatory subdivision with shape constraints for curves. SIAM J. Numer. Anal. 44, 1095-1121 (2006)

27. C. Manni: On shape preserving $C^{2}$ Hermite interpolation. Bit Numerical Mathematics 41(1), 127-148 (2001)

28. M. Marinov, N. Dyn, D. Levin: Geometrically controlled 4-point interpolatory schemes. In: N.A. Dodgson, M.S. Floater and M.A. Sabin (Eds.), Advances in Multiresolution for Geometric Modelling, pp. 301-315, Springer-Verlag (2005)

29. J.-L. Merrien, P. Sablonnière: Monotone and convex $C^{1}$ Hermite interpolants generated by a subdivision algorithm. Constr. Approx. 19, 279-298 (2003)

30. L. Romani: From approximating subdivision schemes for exponential splines to high-performance interpolating algorithms. J. Comput. Appl. Math. 224(1), 383-396 (2009)

31. S. Schaefer, R. Goldman: Non-uniform subdivision for B-splines of arbitrary degree. Comput. Aided Geom. Design 26(1), 75-81 (2009)

32. T.W. Sederberg, J. Zheng, D. Sewell, M. Sabin: Non-uniform recursive subdivision surfaces. Proceedings of the 25 th annual conference on Computer Graphics and Interactive Techniques, 1998, pp. 387-394.

33. J. Warren: Binary subdivision schemes for functions over irregular knot sequences. In: M. Dæhlen, T. Lyche, L.L. Schumaker (Eds.), Mathematical Methods in CAGD III, pp. 543-562, Vanderbilt University Press, Nashville (TN), (1995)

\section{Appendix}

The following expressions define the coefficients of the NULI 6-point scheme.

$$
\begin{aligned}
& a_{i,-6}=-\frac{d_{i-3} d_{i-2}\left(-1+\lambda_{i-2}\right)^{2}\left(d_{i-3}+d_{i-2} \lambda_{i-1}\right)^{2}}{3 d_{i-1}\left(d_{i-2}+d_{i-1}\right)\left(d_{i-3}+d_{i-2}+d_{i-1}\right)\left(d_{i-2}\left(-2+\lambda_{i-2}\right) \lambda_{i-1}+d_{i-3}\left(-1+\lambda_{i-2}\right)\left(1+\lambda_{i-1}\right)\right)}, \\
& a_{i,-5}=-\frac{d_{i-2}^{2}\left(d_{i-3}+d_{i-2} \lambda_{i-1}\right)}{3 d_{i-1}\left(d_{i-2}+d_{i-1}\right)\left(d_{i-3}+d_{i-2}+d_{i-1}\right)}, \\
& a_{i,-4}=\frac{\left(d _ { i - 2 } \left(d_{i-1}^{3}\left(d_{i}+d_{i-1}\right)\left(-1+\lambda_{i-1}\right)^{2} \lambda_{i}^{2}+d_{i-2}^{3}\left(-1+\lambda_{i-1}\right)^{2}\left(d_{i}+d_{i-1}+d_{i} \lambda_{i}\right)\right.\right.}{\left(3 d_{i} d_{i-1}\left(d_{i-2}+d_{i-1}\right)\left(d_{i-3}+d_{i-2}+d_{i-1}\right)\left(d_{i-1}\left(-2+\lambda_{i-1}\right) \lambda_{i}+d_{i-2}\left(-1+\lambda_{i-1}\right)\left(1+\lambda_{i}\right)\right)\right)} \\
& +\frac{d_{i-3}\left(d_{i-1}^{2}\left(d_{i-1}\left(-1+\lambda_{i-1}\right)^{2}+d_{i}\left(-2+\lambda_{i-1}\right) \lambda_{i-1}\right) \lambda_{i}^{2}+d_{i-2}^{2}\left(-1+\lambda_{i-1}\right)^{2}\left(d_{i}+d_{i-1}+d_{i} \lambda_{i}\right)\right.}{\left(3 d_{i} d_{i-1}\left(d_{i-2}+d_{i-1}\right)\left(d_{i-3}+d_{i-2}+d_{i-1}\right)\left(d_{i-1}\left(-2+\lambda_{i-1}\right) \lambda_{i}+d_{i-2}\left(-1+\lambda_{i-1}\right)\left(1+\lambda_{i}\right)\right)\right)} \\
& +\frac{d_{i-3}\left(d_{i-1}\left(d_{i-1}\left(-1+\lambda_{i-1}\right)\left(d_{i-3}+d_{i-2}+d_{i-1}\right)\left(d_{i-1}\left(-2+\lambda_{i-1}\right) \lambda_{i}+d_{i-2}\left(-1+\lambda_{i-1}\right)\left(1+\lambda_{i}\right)\right)\right)\right.}{\left(3 d_{i-1}\right)} \\
& +\frac{\left.d_{i-2} d_{i-1}\left(-1+\lambda_{i-1}\right) \lambda_{i}\left(2\left(d_{i}+d_{i-1}\right)\left(-1+\lambda_{i-1}\right)+d_{i} \lambda_{i-1} \lambda_{i}\right)\right)}{\left(3 d_{i} d_{i-1}\left(d_{i-2}+d_{i-1}\right)\left(d_{i-3}+d_{i-2}+d_{i-1}\right)\left(d_{i-1}\left(-2+\lambda_{i-1}\right) \lambda_{i}+d_{i-2}\left(-1+\lambda_{i-1}\right)\left(1+\lambda_{i}\right)\right)\right)} \\
& +\frac{d_{i-2}^{2} d_{i-1}\left(-1+\lambda_{i-1}\right)\left(d_{i-1}\left(-1+\lambda_{i-1}\right)\left(1+2 \lambda_{i}\right)+d_{i}\left(-1-\lambda_{i}\left(3+\lambda_{i}\right)+\lambda_{i-1}\left(1+\lambda_{i}\right)\left(1+2 \lambda_{i}\right)\right)\right)}{\left(3 d_{i} d_{i-1}\left(d_{i-2}+d_{i-1}\right)\left(d_{i-3}+d_{i-2}+d_{i-1}\right)\left(d_{i-1}\left(-2+\lambda_{i-1}\right) \lambda_{i}+d_{i-2}\left(-1+\lambda_{i-1}\right)\left(1+\lambda_{i}\right)\right)\right)} \\
& +\frac{\left.d_{i-2} d_{i-1}^{2} \lambda_{i}\left(d_{i-1}\left(-1+\lambda_{i-1}\right)^{2}\left(2+\lambda_{i}\right)+d_{i}\left(2\left(1+\lambda_{i}\right)+\lambda_{i-1}\left(-4-5 \lambda_{i}+2 \lambda_{i-1}\left(1+\lambda_{i}\right)\right)\right)\right)\right)}{\left(3 d_{i} d_{i-1}\left(d_{i-2}+d_{i-1}\right)\left(d_{i-3}+d_{i-2}+d_{i-1}\right)\left(d_{i-1}\left(-2+\lambda_{i-1}\right) \lambda_{i}+d_{i-2}\left(-1+\lambda_{i-1}\right)\left(1+\lambda_{i}\right)\right)\right)}, \\
& a_{i,-3}=\frac{d_{i-1}\left(d_{i}+d_{i-1}\right) \lambda_{i}+d_{i-2}\left(d_{i}+d_{i-1}+d_{i} \lambda_{i}\right)}{3 d_{i}\left(d_{i-2}+d_{i-1}\right)}, \\
& a_{i,-2}=\frac{\left(d _ { i - 2 } \left(d_{i}^{3}\left(d_{i}+d_{i+1}\right)\left(-2+\lambda_{i}\right) \lambda_{i+1}^{2}-d_{i-1}^{3}\left(-1+\lambda_{i}\right)^{2}\left(2 d_{i}+d_{i+1}+\left(d_{i}+d_{i+1}\right) \lambda_{i+1}\right)\right.\right.}{\left(3 d_{i} d_{i-1}\left(d_{i-2}+d_{i-1}\right)\left(d_{i}+d_{i+1}\right)\left(d_{i}\left(-2+\lambda_{i}\right) \lambda_{i+1}+d_{i-1}\left(-1+\lambda_{i}\right)\left(1+\lambda_{i+1}\right)\right)\right)} \\
& +\frac{d_{i}^{2} d_{i-1} \lambda_{i+1}\left(\left(d_{i}+d_{i+1}\right)\left(-5+\lambda_{i}+\lambda_{i}^{2}\right)-\left(d_{i+1}\left(1+\left(-3+\lambda_{i}\right) \lambda_{i}\right)+d_{i}\left(2+\lambda_{i}\left(-5+2 \lambda_{i}\right)\right)\right) \lambda_{i+1}\right)}{\left(3 d_{i} d_{i-1}\left(d_{i-2}+d_{i-1}\right)\left(d_{i}+d_{i+1}\right)\left(d_{i}\left(-2+\lambda_{i}\right) \lambda_{i+1}+d_{i-1}\left(-1+\lambda_{i}\right)\left(1+\lambda_{i+1}\right)\right)\right)} \\
& +\frac{\left.-d_{i} d_{i-1}^{2}\left(-1+\lambda_{i}\right)\left(-\left(d_{i}+d_{i+1}\right)\left(2+\lambda_{i}\right)+\left(d_{i+1}\left(-4+\lambda_{i}\right)+3 d_{i}\left(-2+\lambda_{i}\right)\right) \lambda_{i+1}+\left(d_{i}+d_{i+1}\right) \lambda_{i} \lambda_{i+1}^{2}\right)\right)}{\left(3 d_{i} d_{i-1}\left(d_{i-2}+d_{i-1}\right)\left(d_{i}+d_{i+1}\right)\left(d_{i}\left(-2+\lambda_{i}\right) \lambda_{i+1}+d_{i-1}\left(-1+\lambda_{i}\right)\left(1+\lambda_{i+1}\right)\right)\right)} \\
& +\frac{-d_{i-1}\left(-d_{i}^{3}\left(d_{i}+d_{i+1}\right)\left(-3+\lambda_{i}\right) \lambda_{i+1}^{2}+d_{i-1}^{3}\left(-1+\lambda_{i}\right)^{2}\left(2 d_{i}+d_{i+1}+\left(d_{i}+d_{i+1}\right) \lambda_{i+1}\right)\right.}{\left(3 d_{i} d_{i-1}\left(d_{i-2}+d_{i-1}\right)\left(d_{i}+d_{i+1}\right)\left(d_{i}\left(-2+\lambda_{i}\right) \lambda_{i+1}+d_{i-1}\left(-1+\lambda_{i}\right)\left(1+\lambda_{i+1}\right)\right)\right)} \\
& +\frac{d_{i}^{2} d_{i-1} \lambda_{i+1}\left(-\left(d_{i}+d_{i+1}\right)\left(-5+\lambda_{i}+\lambda_{i}^{2}\right)+\left(d_{i+1}\left(3+\left(-5+\lambda_{i}\right) \lambda_{i}\right)+d_{i}\left(4+\lambda_{i}\left(-7+2 \lambda_{i}\right)\right)\right) \lambda_{i+1}\right)}{\left(3 d_{i} d_{i-1}\left(d_{i-2}+d_{i-1}\right)\left(d_{i}+d_{i+1}\right)\left(d_{i}\left(-2+\lambda_{i}\right) \lambda_{i+1}+d_{i-1}\left(-1+\lambda_{i}\right)\left(1+\lambda_{i+1}\right)\right)\right)} \\
& +\frac{\left.\left.d_{i} d_{i-1}^{2}\left(-1+\lambda_{i}\right)\left(-\left(d_{i}+d_{i+1}\right)\left(2+\lambda_{i}\right)+\left(d_{i+1}\left(-4+\lambda_{i}\right)+3 d_{i}\left(-2+\lambda_{i}\right)\right) \lambda_{i+1}+\left(d_{i}+d_{i+1}\right)\left(-1+2 \lambda_{i}\right) \lambda_{i+1}^{2}\right)\right)\right)}{\left(3 d_{i} d_{i-1}\left(d_{i-2}+d_{i-1}\right)\left(d_{i}+d_{i+1}\right)\left(d_{i}\left(-2+\lambda_{i}\right) \lambda_{i+1}+d_{i-1}\left(-1+\lambda_{i}\right)\left(1+\lambda_{i+1}\right)\right)\right)}, \\
& a_{i,-1}=\frac{d_{i}\left(d_{i}+d_{i+1}-d_{i} \lambda_{i+1}\right)+d_{i-1}\left(d_{i}+2 d_{i+1}-\left(d_{i}+d_{i+1}\right) \lambda_{i+1}\right)}{3 d_{i-1}\left(d_{i}+d_{i+1}\right)}, \\
& a_{i, 0}=\frac{-\left(d _ { i + 1 } \left(d_{i}\left(d_{i}+d_{i+1}+d_{i+2}\right)\left(d_{i}+d_{i+1}-d_{i} \lambda_{i+1}\right)^{2} \lambda_{2+i}^{2}+d_{i-1}\left(-d_{i+1}^{3}\left(-2+\lambda_{i+1}\right) \lambda_{i+2}^{2}\right.\right.\right.}{\left(3 d_{i} d_{i-1}\left(d_{i}+d_{i+1}\right)\left(d_{i}+d_{i+1}+d_{i+2}\right)\left(d_{i}+\left(d_{i}+2 d_{i+1}\right) \lambda_{i+2}-\lambda_{i+1}\left(d_{i}+\left(d_{i}+d_{i+1}\right) \lambda_{i+2}\right)\right)\right)} \\
& +\frac{d_{i}^{2}\left(-1+\lambda_{i+1}\right)^{2}\left(-d_{i+2}+\left(d_{i}+d_{i+2}\right) \lambda_{i+2}^{2}\right)+d_{i} d_{i+1}\left(-1+\lambda_{i+1}\right)\left(d_{i}-d_{i} \lambda_{i+1}+\left(d_{i}-d_{i+2}\right)\left(-1+\lambda_{i+1}\right) \lambda_{i+2}\right.}{\left(3 d_{i} d_{i-1}\left(d_{i}+d_{i+1}\right)\left(d_{i}+d_{i+1}+d_{i+2}\right)\left(d_{i}+\left(d_{i}+2 d_{i+1}\right) \lambda_{i+2}-\lambda_{i+1}\left(d_{i}+\left(d_{i}+d_{i+1}\right) \lambda_{i+2}\right)\right)\right)} \\
& +\frac{\left.\left.\left.\left.\left(d_{i+2}\left(-3+\lambda_{i+1}\right)+2 d_{i}\left(-2+\lambda_{i+1}\right)\right) \lambda_{2+i}^{2}\right)+d_{i+1}^{2} \lambda_{i+2}\left(-d_{i}+\left(-2+\lambda_{i+1}\right)\left(-\left(3 d_{i}+d_{i+2}\right) \lambda_{i+2}+d_{i} \lambda_{i+1}\left(-1+2 \lambda_{i+2}\right)\right)\right)\right)\right)\right)}{\left(3 d_{i} d_{i-1}\left(d_{i}+d_{i+1}\right)\left(d_{i}+d_{i+1}+d_{i+2}\right)\left(d_{i}+\left(d_{i}+2 d_{i+1}\right) \lambda_{i+2}-\lambda_{i+1}\left(d_{i}+\left(d_{i}+d_{i+1}\right) \lambda_{i+2}\right)\right)\right)}, \\
& a_{i, 1}=\frac{d_{i+1}^{2}\left(-d_{i+2}+d_{i+1}\left(-1+\lambda_{i+2}\right)\right)}{3 d_{i}\left(d_{i}+d_{i+1}\right)\left(d_{i}+d_{i+1}+d_{i+2}\right)}, \\
& a_{i, 2}=-\frac{d_{i+1} d_{i+2}\left(d_{i+2}-d_{i+1}\left(-1+\lambda_{i+2}\right)\right)^{2} \lambda_{i+3}^{2}}{3 d_{i}\left(d_{i}+d_{i+1}\right)\left(d_{i}+d_{i+1}+d_{i+2}\right)\left(d_{i+2}\left(-2+\lambda_{i+2}\right) \lambda_{i+3}+d_{i+1}\left(-1+\lambda_{i+2}\right)\left(1+\lambda_{i+3}\right)\right)} .
\end{aligned}
$$


Note that the coefficients $a_{i, j}, j=-1, \ldots, 2$ can be obtained from $a_{i, j}, j=-3, \ldots,-6$ by applying the transformation

$$
d_{i+j} \rightarrow d_{i-j-1}, \quad j=-3, \ldots, 2, \quad \lambda_{i+j} \rightarrow 1-\lambda_{i-j+1}, \quad j=-2, \ldots, 3 .
$$

